\title{
Elucidation and Structural Modeling of CD71 as a Molecular Target for Cell-Specific Aptamer Binding
}

\author{
Xiaoqiu Wu ${ }^{\dagger, \nabla}$, Honglin Liu ${ }^{\dagger}, \|, \nabla$, Dongmei Han ${ }^{\dagger, \nabla}$, Bo Peng ${ }^{\dagger}$, Hui Zhang ${ }^{\dagger}$, Lin Zhang ${ }^{\dagger}$, \\ Jianglin $\mathrm{Li}^{\dagger}$, Jing $\mathrm{Liu}^{\ddagger}$, Cheng Cui ${ }^{\dagger}, \perp$, Senbiao Fang ${ }^{\S}$, Min $\mathrm{Li}^{\S}$, Mao $\mathrm{Ye}^{*}, \dagger$, and Weihong \\ $\operatorname{Tan}^{\star}, \dagger, \perp, \#$ \\ †Molecular Science and Biomedicine Laboratory (MBL), State Key Laboratory of Chemo/ \\ Biosensing and Chemometrics, College of Biology, College of Chemistry and Chemical \\ Engineering, Aptamer Engineering Center of Hunan Province, Hunan University, Changsha, \\ Hunan 410082, China \\ ‡Molecular Biology Research Center, School of Life Sciences, Central South University, \\ Changsha, Hunan 410078, China
}

$\S$ School of Information Science and Engineering, Central South University, Changsha, Hunan 410078, China

"School of Food and Biological Engineering, Hefei University of Technology, Hefei, Anhui 230009, China

${ }^{\perp}$ Department of Chemistry and Department of Physiology and Functional Genomics, Center for Research at the Bio/Nano Interface, Health Cancer Center, UF Genetics Institute, McKnight Brain Institute, University of Florida, Gainesville, Florida 32611, United States

\#Institute of Molecular Medicine (IMM), Renji Hospital, Shanghai Jiao Tong University School of Medicine, and College of Chemistry and Chemical Engineering, Shanghai Jiao Tong University, Shanghai 200240, China

\section{Abstract}

Pancreatic cancer is a highly lethal malignancy associated with tissues of the pancreas. Early diagnosis and effective treatment are crucial to improving the survival rate of patients with pancreatic cancer. In a previous study, we employed the cell-SELEX strategy to obtain an ssDNA aptamer termed XQ-2d with high binding affinity for pancreatic cancer. Here, we first identify CD71 as the XQ-2d-binding target. We found that knockdown of CD71 abolished the binding of $\mathrm{XQ}-2 \mathrm{~d}$ and that the binding affinity of XQ-2d is associated with membrane-bound CD71, rather than total CD71 levels. Competitive analysis revealed that XQ-2d shares the same binding site on CD71 with transferrin (Tf), but not anti-CD71 antibody. We then used a surface energy transfer (SET) nanoruler to measure the distance between the binding sites of XQ-2d and anti-CD71

\footnotetext{
*Corresponding Authors: goldleaf@hnu.edu.cn, tan@chem.ufl.edu.

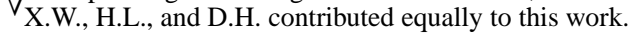

Supporting Information

The Supporting Information is available free of charge on the ACS Publications website at DOI: 10.1021/jacs.9b03720.

Representative MS/MS of tryptic peptide from CD71, binding mode prediction between CD71 and XQ-2d, and cell viability (PDF)

The authors declare no competing financial interest.
} 
antibody, and it was about $15 \mathrm{~nm}$. Furthermore, we did molecular dynamics simulation to clarify that the spatial structure of XQ-2d and Tf competitively binding to CD71. We also engineered XQ-2d-mediated targeted therapy for pancreatic cancer, using an XQ-2d-based complex for loading doxorubicin (Dox). Because CD71 is overexpressed not only in pancreatic cancer but also in a variety of tumors, our work provides a systematic novel way of studying a potential biomarker and also promising tools for cancer diagnosis and therapy, opening new doors for effective cancer theranostics.

\section{Graphical Abstract}

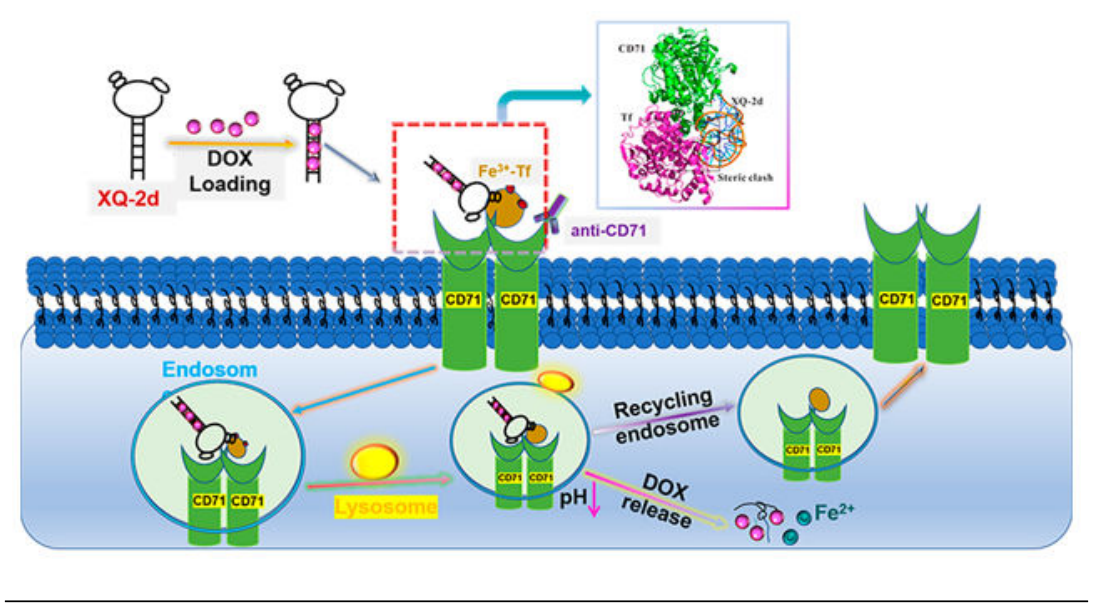

\section{INTRODUCTION}

Pancreatic cancer is caused by the abnormal and uncontrolled growth of cells in pancreatic tissues. It is a highly lethal malignancy and the third leading cause of cancer deaths in the United States. ${ }^{1}$ The most common type of pancreatic cancer is pancreatic ductal adenocarcinoma, accounting for $>90 \%$ pancreatic cancers. ${ }^{2}$ Studies have shown that the median survival of pancreatic cancer patients without surgical resection is $2-8$ months and that only $\sim 6 \%$ of all patients survive more than 5 years after diagnosis. ${ }^{3}$ Poor prognosis stems from a lack of effective therapy and late diagnosis when the cancer has already metastasized to distant organ sites. ${ }^{3}$ Despite recent progress in pancreatic cancer therapy, the survival rate has not significantly improved. Therefore, early diagnosis and effective treatment are crucial to improving the survival rate.

One tool in the search for solutions is the aptamer molecule. Aptamers are single-stranded DNA or RNA oligonucleotides with high affinity and selectivity for various targets, ranging from small molecules and ions to complex mixtures such as cells. ${ }^{4}$ Aptamer-target binding is achieved mainly from structural compatibility, stacking of aromatic rings, electrostatic and van der Waals interactions, and hydrogen bonding, or some combination thereof. ${ }^{5}$ Functionally, aptamers are similar to protein antibodies. Yet aptamers exhibit unique chemical and biological characteristics that differ from those of conventional antibodies, based on their oligonucleotide properties, including ease of chemical synthesis, high chemical stability, low molecular weight, lack of immunogenicity, rapid tissue penetration, 
low toxicity, and ease of modification and manipulation. ${ }^{6}$ Therefore, aptamers have high potential for diagnosis, biomarker discovery, therapy, and drug delivery in the cancer field. ${ }^{7,8}$

In general, a specific aptamer is obtained by the in vitro SELEX (Systematic Evolution of Ligands by EXponential enrichment) process from a random oligonucleotide library. To generate cell-specific aptamers, a newer variant technology, cell-SELEX, has been developed using intact living cells as the target. ${ }^{9-11}$ Because all molecular targets on the cell surface retain their native states, including native folding structure and post-translational modification, during the SELEX process, aptamers selected against whole cells will bind to target molecules in their native folded conformation. Moreover, cell-specific aptamers can be used as probes to identify the specific binding target, thus contributing to the isolation of diseased-related targets and facilitating the discovery of clinically important biomarkers for disease diagnosis and targeted therapy. On the basis of the cell-SELEX selection strategy, several cell-specific aptamer-binding targets have been identified and used in cancer diagnosis and therapy, such as PTK7 for acute lymphoblastic leukemia, ${ }^{12}$ IGHM for Burkitt's lymphoma, ${ }^{13}$ tenascin-C for glioblastoma, ${ }^{14}$ and STIP1 for ovarian cancer. ${ }^{15}$

In a previous study, we employed cell-SELEX strategy to obtain an ssDNA aptamer, termed XQ-2d, which showed high binding affinity for pancreatic ductal adenocarcinoma. ${ }^{16}$ In the present study, we identified CD71, highly expressed in a number of solid and hematologic cancers, as the binding target of XQ-2d, and revealed the possible binding site of XQ-2d on CD71. Furthermore, we explored XQ-2d-mediated therapy for pancreatic cancer based on cellular internalization. Because CD71 is overexpressed not only in pancreatic cancer but also in a variety of tumors, our work provides a promising tool for cancer diagnosis and therapy and opens the door for developing new strategies for efficiently controlling cancer.

\section{RESULTS}

\section{Identification of $\mathrm{XQ}-2 \mathrm{~d}-$ Binding Proteins through Mass Spectrometry Analysis.}

In a previous study, we employed the cell-SELEX strategy to obtain an ssDNA aptamer, termed XQ-2d, which showed high binding affinity for pancreatic ductal adenocarcinoma PL45 cells with dissociation constants in the nanomolar range. Meanwhile, we also demonstrated that XQ-2d specifically recognized PL45 xenograft in nude mice and human pancreatic ductal adenocarcinoma tissues. To investigate what type of target molecules are bound by XQ-2d, PL45 cells were treated with Proteinase K or trypsin for $10 \mathrm{~min}$, followed by incubation with XQ-2d. Intriguingly, XQ-2d completely lost its ability to bind PL45 cells after enzymatic treatment as compared to the nontreatment group (Figure 1A). This result indicated that molecules of the XQ-2d-binding target on the cell surface had been fully removed by enzymes, implying that the binding targets of XQ-2d were probably membranebound proteins.

On the basis of the above result, we next focused on the identification of membrane proteins bound by XQ-2d. To this end, total membrane proteins of PL45 cells were extracted and incubated with biotinylated XQ-2d or library, respectively. The binding complex was then separated using streptavidin-coated magnetic beads and analyzed through SDS-PAGE gel. Intriguingly, one protein band with relative molecular mass of $\sim 100 \mathrm{kDa}$ was specifically 
present among the associated factors obtained from the XQ-2d column, but not from the library column or the control column (Figure 1B). The characteristic protein band was then digested and submitted to LC-MS/MS QSTAR analysis. As shown in Table S1, the MS results produced a list of protein hits. Among these candidates, CD71 ranked first with the highest score and the maximum content. Because CD71, also known as the transferrin receptor, is a type II membrane glycoprotein and has frequent high expression in pancreatic cancer, we focused our attention on CD71 as a potential binding target of XQ-2d.

\section{Validation of CD71 as a target for XQ-2d.}

To confirm the result from mass spectrometry, we first examined the location of XQ-2d and CD71 on PL45 cells. To accomplish this, PL45 cells were respectively incubated with antiCD71 antibody conjugated to phycoerythrin (PE)- and fluorescein isothiocyanate (FITC)labeled XQ-2d. An intense yellow color was observed in merged images, indicating the spatial colocalization between XQ-2d and CD71. Notably, florescence signals from XQ-2d, or CD71, were detected from both the cell surface and the cell body (Figure 2A). We speculated that CD71 has a strong ability to internalize into the cells. To determine whether XQ-2d interacts with CD71, membrane proteins of PL45 cells were extracted and incubated with agarose beads, agarose-conjugated XQ-2d, or agarose-conjugated library, respectively. The pull-down mixture contained proteins that were then subjected to Western blot analysis using CD71-specific antibody. As expected, CD71 was easily pulled down by XQ-2d (Figure 2B, lane 4), but not by agarose beads or library (Figure 2B, lane 2 and lane 3), demonstrating the interaction between XQ-2d and CD71. Furthermore, we performed a lossof-function analysis using two independent CD71-specific small interfering RNAs (siRNAs). As shown in Figure 2C, siRNAs produced a significant knockdown effect of CD71 as compared to the control group. Consistent with this, the binding of XQ-2d to PL45 cells was profoundly abolished by siRNAs (Figure 2D). Analysis of the binding affinity by ELISA revealed that the equilibrium dissociation constant $\left(K_{\mathrm{d}}\right)$ of XQ-2d against CD71 was $50.5 \pm 27.3 \mathrm{nM}$ (Figure 2E). In addition, the $K_{\mathrm{d}}$ of anti-CD71 antibody for PL45 cells was approximately $635 \pm 29.65 \mathrm{pM}$ (Figure S2). Taken together, our data demonstrate that CD71 is the binding target of XQ-2d on PL45 cells.

\section{XQ-2d-Binding of CD71 on the Cell Surface.}

XQ-2d binds to PL45 cells through recognizing CD71 protein. We therefore asked if the binding affinity of XQ-2d is associated with the expression levels of CD71. To test this possibility, total protein from four different types of cell lines was extracted, and the expression of CD71 was analyzed by Western blot. We found that CD71 exhibited the highest expression in human lung cancer cell line H460, followed by human embryonic kidney cell line HEK293 and human colon cancer cell line HCT116, but that PL45 cells showed the lowest expression of CD71 (Figure 3A and B). Unexpectedly, XQ-2d showed a strong binding to PL45 and H460 cells, whereas no significant binding to HEK293 and HCT116 was observed (Figure 3C). This result was inconsistent with the expression levels of CD71 in the above four types of cell lines. Because the binding target molecules of $\mathrm{XQ}-2 \mathrm{~d}$ are membrane proteins, we hypothesized that XQ-2d could be associated with membrane-bound CD71, not total CD71 levels. To address this, cells were incubated with PE-labeled antibody that recognizes the extracellular region of CD71. As shown in Figure 
3D, a strong fluorescence shift was observed in PL45 and H460 cells, but not HEK293 and HCT116 cells. Meanwhile, relative fluorescence intensity in PL45 and H460 cells was far greater than that in HEK293 and HCT116 cells (Figure 3E), which is consistent with the XQ-2d-binding pattern. When PL45 cells were pretreated with Dynasore, an inhibitor of endocytosis, the fluorescence signal from XQ-2d was found to have transferred from the cell body to the cell surface (Figure 3F), indicating that the internalization of XQ-2d is dependent on the endocytosis of CD71. Collectively, these results suggest that membranebound CD71 is the target of XQ-2d.

\section{XQ-2d Shares the Similar Binding Site on CD71 with Transferrin, but Not Anti-CD71 Antibody.}

Because transferrin (Tf) is a natural ligand of CD71, a competition assay was performed by flow cytometry. Different concentrations of unlabeled Tf were incubated with FITC-labeled XQ-2d. Interestingly, flow cytometry results showed that XQ-2d gradually lost its binding to PL45 cells as the concentration of Tf (Figure 4A). To further confirm the blocking effect of Tf, the binding of XQ-2d to PL45 cells was examined in the absence or presence of excess Tf by confocal microscopy. As expected, an intense fluorescence signal was observed in PL45 cells only when incubated with FITC-labeled XQ-2d, but not when incubated with Tf followed by XQ-2d (Figure 4B). These results indicated competition between XQ-2d and Tf, implying that the same binding site on CD71 was shared by XQ-2d and transferrin.

On the basis of the above results, we next asked whether XQ- $2 \mathrm{~d}$ also shares the same binding site with anti-CD71 antibody. To this end, excess XQ-2d was incubated with PL45 cells, followed by PE-labeled anti-CD71 antibody. Flow cytometry results showed no obvious change in the binding of anti-CD71 antibody to PL45 cells (Figure 4C). Likewise, Tf had no influence on anti-CD71 antibody binding (Figure 4D). A binding assay of XQ-2d, Tf, and anti-CD71 antibody to PL45 cells was then performed using confocal microscopy. Consistent with the flow cytometry results, an intense fluorescence signal could be detected when PL45 cells were incubated with PE-labeled anti-CD71 antibody in the absence or presence of XQ-2d or Tf, indicating that neither XQ-2d nor Tf abrogated anti-CD71 antibody binding (Figure 4E). To further investigate the possible cobinding of XQ-2d and antibody on CD71, FITC-labeled XQ-2d or PE-labeled anti-CD71 antibody was incubated with PL45 cells. The fluorescence signal dot plot from flow cytometry analysis is shown in Figure 4F. Interestingly, fluorescence signals from the FITC and PE channels displayed a linear relationship, indicating that the different sites of the extracellular domain of CD71 were simultaneously bound by XQ-2d and antibody.

\section{A SET Nanoruler for Measuring Binding Site Distances on PL45 Cell Surfaces.}

Previously, our group developed a SET nanoruler to determine the distance between binding sites of an aptamer and antibody on a live cell surface. ${ }^{17}$ Herein, we constructed a similar SET nanoruler to estimate the possible binding site of XQ-2d on CD71. PE-labeled antiCD71 antibody and thiolated XQ-2d were modified to carry a red fluorophore and a gold nanoparticle (GNP), respectively, to each of the binding sites on the PL45 cell membrane. The GNPs were functionalized with excess thiol-modified XQ-2d on their surfaces, and the anti-CD71 antibody carried a red PE fluorophore. The binding of XQ-2d and anti-CD71 
antibody to their individual binding sites around receptor CD71 would bring fluorophore and GNPs into close proximity. In principle, GNPs with excellent fluorescence quenching capability could act as the energy acceptor and effectively induce energy transfer from the PE fluorophore to the metal surface (Figure 5A).

Our previous work has demonstrated that the distance, $R$, between two binding sites on the receptor could be estimated on the basis of the distance from the PE fluorophore to the center of the GNP. In a simplified model, $\mathrm{R}$ is equal to the sum of the distance, $r$, from the fluorophore to the GNP surface and the radius of the particle, $d / 2$, with

$$
R=r+d / 2
$$

Hence, varying GNP size ( $d$ ) accordingly changes the distance from the fluorophore to the GNP surface $(r)$. We assumed that the statistical analysis of one million cells each time would balance out the variations in GNP position around the aptamer binding site.

Therefore, we could manipulate the distance from the PE molecule to the metal surface by controlling the GNP size, thereby allowing us to determine the relationship between GNP size and the energy transfer efficiency.

When using the SET nanoruler for distance measurements, it is important that both binding sites be under saturation binding conditions by the corresponding ligands. Therefore, to determine the saturation concentration, quantitative binding experiments were carried out for the PE-labeled anti-CD71 antibody and the XQ-2d-modified GNP with the diameter of 13.3 $\mathrm{nm}$ (Figure 5B) on their own binding sites on the PL45 cell membrane. The results indicated a $200 \mathrm{nM}$ saturation concentration for PE-labeled anti-CD71 at the antibody binding sites; for XQ-2d-GNPs at $13.3 \mathrm{~nm}$, a quantitative binding curve was plotted for concentrations ranging from 0 to $15 \mathrm{nM}$ by incubating the aptamer-GNP probe with the same number of cells (106). Figure 5B shows a plateau with a saturation concentration of $8 \mathrm{nM}$. All following experiments were carried out using these concentrations to ensure the consistency of the same saturation conditions.

Similarly, the SET nanoruler was constructed with different sizes of GNPs from 10.0 to 25.0 $\mathrm{nm}$ (Figure 5C). Flow cytometry measurements produced histograms to quantitatively monitor the energy transfer from the PE fluorophore to the GNP surface. As compared to the binding of PE-labeled anti-CD71 alone, the presence of the XQ-2d-GNP probe on the aptamer binding sites resulted in an obvious fluorescence quenching of the PE fluorophore, and more quenching occurred when GNP diameters increased, because a large GNP brings its surface closer to the PE fluorophore. A significant fluorescence decrease from the PElabeled anti-CD71 was observed as the GNP size increased from $10.0 \mathrm{~nm}$ (94.2\% FL intensity as compared to PE-labeled anti-CD71 alone) to $18.4 \mathrm{~nm}$ (76.4\% FL intensity as compared to PE-labeled anti-CD71 alone). When the GNP diameter exceeded $18 \mathrm{~nm}$, the quenching showed similar levels and reached a plateau, indicating that the GNP surface had reached PE molecules and produced the maximum fluorescence quenching, while GNPs with diameters exceeding $18 \mathrm{~nm}$ no longer caused fluorescence quenching. Control experiments were also carried out using $5^{\prime}$-thiolated library DNA sequence (Lib)-modified 
GNPs with different sizes, and, as shown in Figure 5C, the fluorescence quenching efficiency of the Lib-capped GNPs with diameter of $18.4 \mathrm{~nm}$ was $90.8 \%$. Therefore, we can infer that the fluorescence quenching from XQ2d-GNPs on the aptamer binding sites did not result from the effects of nonspecific steric hindrance.

Figure 5D shows a plot of the quenching efficiency $(\Phi)$ versus different GNP diameters $(d)$, and the results show a plateau after the GNP diameter reached $\sim 18 \mathrm{~nm}$. This distancedependent quenching behavior can be fitted with the SET model proposed by Yun et al., ${ }^{18}$ as

$$
\varnothing=\frac{1}{1+\left(r / r_{0}\right)^{4}}
$$

where $r_{0}$ is the distance at which a fluorophore displays $50 \%$ probability of both energy transfer and spontaneous emission. Combined with eq 1 , we have the reconfigured equation:

$$
\left(\frac{1}{\varnothing}-1\right)^{1 / 4}=-\frac{d}{2 r_{0}}+\frac{R}{r_{0}}
$$

By assigning $Y=(1 / \Phi-1)^{1 / 4}$ and $X=d$, we obtained a simplified eq 4 , as

$$
Y=-\frac{X}{2 r_{0}}+\frac{R}{r_{0}}
$$

Hence, a relationship between $Y$ and $X$ was plotted, as shown in Figure 5E, according to the data in Figure $5 \mathrm{C}$. According to previous reports, $r_{0}$ values vary considerably for large GNPs (>20 nm), while a very similar $r_{0}$ value is obtained for GNP sizes smaller than $20 \mathrm{~nm}$. We assume $r_{0}$ is a constant in this range, as shown in our previous work. ${ }^{17}$ According to the redline fit in Figure 5E, by using the data of three GNP sizes (10.0, 13.3, and $16.2 \mathrm{~nm}$ ), we obtained the calculated values $r_{0}=5.09 \mathrm{~nm}$ and $R=15.2 \mathrm{~nm}$.

Moreover, according to the blueline fit, by using the data of four GNP sizes $(10.0,13.3$, 16.2 , and $18.4 \mathrm{~nm}$ ), we obtained the calculated values $r_{0}=5.99 \mathrm{~nm}$ and $R=16.8 \mathrm{~nm}$.

Thus, the distance between the binding sites of XQ-2d aptamer and anti-CD71 antibody is in the range of 15-17 nm, which is greater than the detection limit for FRET. Because $r_{0}$ values vary so much, especially for large particles, we reasoned that the redline fit would be more reliable; that is, the distance between the two binding sites, $R$, is considered to be about 15 $\mathrm{nm}$. Overall, then, this SET nanoruler provides an evidence-based estimation of the distance between aptamer binding sites in cellular systems that other methods, like FRET or gas chromatography mass spectrometry (GC/MS), cannot provide. 


\section{Identification of XQ-2d and CD71 Binding by Computational Modeling Analysis.}

In a blind docking process, a total number of 2000 of conformations were extracted and ranked by docking energy value. The 200 conformations with the lowest binding energy were used to predict the protein-aptamer binding spots, and the amino acid residues in the target protein CD71 that interact with the selected aptamer XQ-2d conformations were counted to predict the protein functional site. Multiple conformation superposition results analysis indicated that the binding sites between CD71 and XQ-2d were relatively concentrated. Fourteen potential binding spots with relatively high ratio $(r>0.4)$ were mainly selected as XQ-2d binding residues for further precise docking (Figure S3A). Figure S3B displays the complex structure with the lowest binding score from the precise docking process. Detailed analysis showed that XQ-2d could bind tightly with all 14 residues, listed as follows: S638, Q640, K673, Y683, P688, Y689, S691, K693, A736, W740, N747, V753, W754, and N758, and the conformation of CD71-XQ-2d from precise docking could be extracted for further analysis.

The results obtained from the above precise docking analysis provoked us to explore the dynamic behavior of CD71-XQ-2d complexes. A total of $10 \mathrm{~ns}$ simulation time was conducted, and Molecular Dynamics (MDs) trajectory was employed for extracting the refined binding model (Figure 6A). Obviously, the binding interface was mainly located at two regions, part a and part b. Sixteen amino acids of protein CD71 that formed important interactions with XQ-2d were identified as key residues. Polar interactions were mainly involved in stabilizing the complex via nonbonded electrostatic interactions. One intensive hydrogen-bond interaction system was formed at the interface between CD71 and XQ-2d. Two schematic diagrams for each region are shown in Figure 6B and C, and eight nucleic acid bases participated in the formation of an H-bond network with 16 residues of CD71.

Besides polar interactions, the hydrophobic interaction also played a necessary role in complex stability. Considering the benzene ring on Y683 and the carbochain on L471, both make hydrophobic contact with aptamer base R5 (Figure 6B). According to the hydrogen bonds and the complementarity of hydrophobic/hydrophilic properties on CD71 and XQ-2d molecular surface, 16 amino acids were identified as key residues (Figure 6) that drive the interaction and stabilize the CD71-XQ-2d complex. The structure of the complex between CD71 and Tf was downloaded from the RCSB PDB databank. Conformation superposition of crystal structures of CD71, Tf, and XQ-2d showed that XQ-2d possessed an obvious steric clash with the natural Tf (Figure 6D). Thus, our results provide molecular insights into the CD71 binding sites for XQ-2d and evidence that the spatial structure of XQ-2d and Tf competitively binds to CD71.

\section{Construction of XQ-2d-sd3 for Drug Delivery.}

Previous studies show that $\mathrm{Tf}$ supports cell proliferation by supplying iron for DNA synthesis. Given that XQ-2d and Tf competitively bind to CD71 on the surface of PL45 cells, we therefore asked if XQ-2d would affect cell proliferation. To make this determination, different concentrations of XQ-2d were incubated with PL45 cells and HEK293 cells. However, XQ-2d did not exhibit any significant effect on the proliferation of PL45 cells or HEK293 cells (Figure S4). 
Dox is an FDA-approved, effective chemotherapeutic drug against most cancer types, and it can intercalate within the DNA strand due to the presence of flat aromatic rings, preferentially binding to double-stranded $5^{\prime}-\mathrm{GC}-3^{\prime}$ or $5^{\prime}-\mathrm{CG}-3^{\prime}$ sequences. To load more Dox molecules into XQ-2d for drug delivery, a linker tail was added to the $5^{\prime}$-end of XQ-2d, which self-assembled into the GC-enriched XQ-2d-sd3 by incubating with sd3 generated by sd 1 and sd2 via complementary base pairing. The schematic design is shown in Figure 7A. Agarose gel electrophoresis verified the correct assembly of XQ-2d-sd3 (Figure 7B). To test whether XQ-2d-sd3 would maintain its binding ability, PL45 and HEK293 cells were incubated with library, XQ-2d, sd3, and XQ-2d-sd3, respectively. As shown in Figure 7C, both XQ-2d-sd3 and XQ-2d, but not library or sd3, bound to PL45 cells. In contrast, all sequences failed to bind to HEK293 cells (Figure 7D). This result demonstrated that XQ-2dsd3 still retains its selective affinity for PL45 cells. Internalization is very important for targeted drug delivery. To investigate the internalization capacity of XQ-2d-sd3, PL45 cells were incubated with Cy5-labeled XQ-2d-sd 3 at $37{ }^{\circ} \mathrm{C}$ for $2 \mathrm{~h}$. A significant fluorescent signal originated from inside the cells, clearly indicating that XQ-2d-sd3 had entered cells and revealing that XQ-2d-sd3 can be internalized into PL45 cells (Figure 7E).

\section{XQ-2d-Mediated Targeted Therapy for Pancreatic Cancer.}

It is well-known that the intercalation of Dox into DNA will quench the fluorescence signal of Dox. Therefore, we next utilized the fluorescence properties of Dox to evaluate the Doxloading capacity of XQ-2d-sd3. As shown in Figure 8A, the fluorescence of Dox was gradually decreased as the concentration of XQ-2d-sd3 increased. The fluorescence of Dox was almost completely quenched when the XQ-2d-sd3/Dox molar ratio reached 1:15, suggesting that one molecule of XQ-2d-sd3 can conjugate at least 15 Dox molecules. Dox release was also investigated using confocal microscopy. PL45 cells and HEK293 cells were incubated with free Dox and chimeras (XQ-2d-sd3-Dox), respectively. Consistent with previous studies, the red fluorescence from Dox was found in both PL45 cells and HEK293 cells after incubation with free Dox. However, in the group treated with chimeras, the red fluorescence from DOX occurred only in PL45 cells, but not in HEK293 cells, indicating that chimeras selectively deliver Dox to PL45 cells (Figure 8B and C). Furthermore, the concentrations required to inhibit cell growth by $50 \%\left(\mathrm{IC}_{50}\right)$ values of free Dox determined by CCK-8 assay for PL45 and HEK293 cells were 12.5 and $2.92 \mu \mathrm{M}$, respectively (Figure 8D). To examine whether the selective delivery of Dox to PL45 cells would result in targeted cytotoxicity, PL45 and HEK293 cells were incubated with XQ-2d-sd3, free Dox ( $\mathrm{IC}_{50}$ concentration), and chimeras with $\mathrm{IC}_{50}$ concentration of Dox for $48 \mathrm{~h}$, respectively. The result showed that free Dox had similar cytotoxicity in PL45 and HEK293 cells, whereas chimeras significantly reduced cytotoxicity in HEK293 cells (Figure 8E). Taken together, these data suggest that XQ-2d-based chimeras have the potential for targeted therapy through the selective delivery of cytotoxic agents.

\section{DISCUSSION}

A biomarker is defined as a biological molecule, including DNA, RNA, protein, peptide, and chemically modified biomolecule, able to reflect a normal or abnormal biological state in an organism. A cancer biomarker can be used to identify an early-stage cancer and measure risk 
of cancer progression or potential response to therapy. ${ }^{19}$ Aptamers generated by cell-SELEX facilitate the discovery of bio-markers,${ }^{20}$ which are based on unknown molecular differences between disease cells and normal cells. Identification of molecular differences contributes to the discovery of biomarkers. Using cell-SELEX, we obtained an ssDNA aptamer, XQ-2d, with high affinity and specificity for pancreatic ductal adenocarcinoma. In this study, the target of XQ-2d-binding was further identified as CD71. It was found that CD71 is overexpressed in a variety of human cancers. ${ }^{21-23}$ Multiple studies have shown that CD71 significantly participates in pancreatic cancer growth and survival, ${ }^{24,25}$ making it a potential marker of malignant transformation in the pancreas. ${ }^{26,27}$ Thus, our work provides strong support for the cell-SELEX-based approach in discovering potential disease biomarkers.

CD71 is a type II transmembrane glycoprotein that consists of a transferrin-binding Cterminal domain, a short $\mathrm{N}$-terminal domain, and a single transmembrane domain. ${ }^{28}$ Two identical CD71 subunits are linked as an integral membrane homodimer that can bind ironladen transferrin, and the resulting complex is internalized through endocytosis mediated by clathrin. Interestingly, we found that the binding affinity of XQ-2d to cells was not related to total CD71 levels, but rather depended on membrane-bound CD71. In response to excess Tf, the binding of XQ-2d to cells was completely abolished, indicating that XQ-2d binds to the extracellular region of CD71 and shares similar binding sites with Tf. Because the extracellular domain of CD71 binds to one molecule of transferrin per subunit, ${ }^{29}$ it would be interesting to test if the same amount of XQ-2d could also mediate the binding to CD71. Furthermore, we identified the CD71 binding sites for XQ-2d and measured the distance between the binding sites of XQ-2d and anti-CD71 antibody on CD71, thus establishing a sound scientific foundation to further clarify the binding mechanism of XQ-2d.

Iron plays critical roles in various physiological and pathological processes. The uptake of Tf-bound iron through CD71 is the main pathway for iron entry into cells. ${ }^{30}$ In the last several decades, the overexpression of CD71 has been found in various cancers, mainly to meet the iron requirement of cancer cell proliferation. ${ }^{31}$ Accumulating evidence has demonstrated that CD71 enhances malignant transformation of cancer cells. ${ }^{26,32}$ Given the association of CD71 and cancer, we hypothesized that CD71-bound XQ-2d could affect the biological function of cancer cells. However, XQ-2d did not exhibit any significant effect on the proliferation of PL45 cells or HEK293 cells. Because 85-95\% of internalized CD71 follows the recycling pathway back to the cell surface, ${ }^{32}$ we reasoned that XQ-2d could be rapidly consumed in this process. Therefore, although XQ-2d competes with Tf for CD71, the proliferation is unaffected. However, further studies are needed to fully understand the internalization of XQ-2d.

CD71-mediated endocytosis is the main route of cellular iron uptake, which has been exploited for the site-specific delivery of anticancer drugs, proteins, and therapeutic genes into cancer cells with overexpressed CD71. Considering the specific binding of XQ-2d to CD71, we constructed XQ-2d-sd3 to load DOX for targeted delivery to pancreatic cancer cells. Interestingly, Dox could be selectively delivered into PL45 cells via XQ-2d-sd3-DOX chimeras, resulting in a significant inhibition of proliferating PL45 cells, indicating that XQ-2d possesses potential as a targeting moiety. Currently, strategies for the targeting of therapeutic agents via CD71 to malignant cells mostly utilize its natural ligand Tf or 
monoclonal antibodies. However, we have discovered the unique advantages of XQ-2d as an aptamer, which will afford a new direction in the development of XQ-2d-based strategies for cancer-targeted therapy.

\section{MATERIALS AND METHODS}

\section{Cell Lines and Reagents.}

Human pancreatic ductal adenocarcinoma cell line PL45 was obtained from the American Type Culture Collection (ATCC). Lung cancer cell line H460, colon carcinoma cell line HCT116, and human embryonic kidney epithelial cell line HEK293 were all obtained from the cell bank of Xiangya Hospital (Changsha, China). All cell lines were cultured at $37{ }^{\circ} \mathrm{C}$ humid atmosphere with 5\% CO2. PL45 cells and HEK293 cells were cultured in DMEM medium supplemented with $10 \%$ fetal bovine serum (FBS, Gibco). H460 cells and HCT116 cells were cultured in RPMI-1640 medium supplemented with 10\% FBS. Rabbit antihuman CD71 monoclonal antibody (\#13113) used for Western blot was purchased from Cell Signaling Technology. PE mouse IgG (555574) and PE mouse anti-CD71 (555537) antibody for flow cytometry were purchased from BD Biosciences. Human holo-transferrin (hTf, T4132) protein and Dox (D1515) were obtained from SIGMA.

\section{DNA Sequences and Buffers .}

Library , NNNNNNNNGGTTAGGGGCTGCTGGCCAGATACTCA-

GATGGTAGGGTTANNNNNNNN. XQ-2d, ACTCATAGGGT-

TAGGGGCTGCTGGCCAGATACTCAGATGGTAGGGTTAC-TATGAGC. XQ-2d+linker, TTCGTTCGTCACTGCCTGTTTTT-

TACTCATAGGGTTAGGGGCTGCTGGCCAGATACTCA-

GATGGTAGGGTTACTATGAGC . S 1 , CTTCCAGACTAACAACAGA. S2, CAGGCAGTGACGAAC-GAATCTGTTGTTAGTCTGGAAG.

All sequences were purchased from Sangon Biotech Co. Ltd. (Shanghai, China). Washing buffer was prepared with Dulbecco's phosphate-buffered saline (D-PBS) supplemented with $4.5 \mathrm{~g} / \mathrm{L}$ of glucose and $5 \mathrm{mM}$ of $\mathrm{MgCl}_{2}$. Binding buffer was prepared with D-PBS supplemented with $4.5 \mathrm{~g} / \mathrm{L}$ of glucose, $5 \mathrm{mM}$ of $\mathrm{MgCl} 2,0.1 \mathrm{mg} / \mathrm{mL}$ of yeast tRNA, and 1 $\mathrm{mg} / \mathrm{mL}$ of BSA.

\section{Flow Cytometric Analysis.}

To analyze the binding ability of aptamer or anti-CD71, $250 \mathrm{nM}$ of FITC-XQ-2d or PElabeled anti-CD71 was prepared in triplicate and incubated with the target cells in $200 \mu \mathrm{L}$ of binding buffer at $4{ }^{\circ} \mathrm{C}$ for $1 \mathrm{~h}$. After incubation, nonbound aptamers or antibody was washed out and then resuspended cells in $500 \mu \mathrm{L}$ of binding buffer. BD FACSVerse flow cytometer was used to measure the fluorescence intensity of each sample.

\section{Western Blot.}

The cultured cells were rinsed with phosphate-buffered saline (PBS) and were lysed in RIPA buffer with protease inhibitor cocktail. The protein concentrations were determined by protein assay reagent (Bio-Rad). Protein samples (30 ug/well) were used for electrophoresis 
in SDS/10\% polyacrylamide gels. Proteins then were electrophoretically transferred to 0.22 $\mu \mathrm{m}$ Nitrocellulose (NC) membranes by the Western blot transfer system (Bio-Rad) for $1.5 \mathrm{~h}$ at $300 \mathrm{~mA}$. The NC membranes were washed with TBST (Tris-buffered saline containing $0.5 \%$ Tween 20) and blocked with 5\% BSA for $1 \mathrm{~h}$ at room temperature. Thereafter, the membranes were washed three times with TBST and incubated with rabbit polyclonal primary antibody specific to CD71 diluted 1:1000 overnight at $4{ }^{\circ} \mathrm{C}$. After being washed, membranes were incubated with goat anti-rabbit HRP-conjugated secondary antibody (Life Technologies), diluted 1:1000 for $1 \mathrm{~h}$ at room temperature, followed by detection using ChemiDoc XRS+ (Bio-Rad).

\section{Determination of Target Type.}

To determine the target type of XQ-2d, flow cytometer was used to measure the fluorescence as discussed before. First, we detached PL45 cells with $0.1 \mathrm{mg} / \mathrm{mL}$ Proteinase $\mathrm{K}$ or $0.25 \%$ trypsin-EDTA, respectively. After being washed three times with D-PBS, $3 \times 10^{5}$ cells were incubated with $250 \mathrm{nM}$ of FITC-labeled ss-DNA library or XQ-2d in $200 \mu \mathrm{L}$ of binding buffer at $4{ }^{\circ} \mathrm{C}$ for $1 \mathrm{~h}$. After resuspended cells were washed in $500 \mu \mathrm{L}$ of binding buffer, the mean fluorescence was analyzed by a flow cytometer (BD FACSVerse flow cytometer) with FlowJo (v7.0) software.

\section{Mass Spectrometry Analysis.}

Because the modification of biotin at the 5-terminal of XQ-2d can decrease binding efficiency, we added six T bases between biotin and the XQ-2d sequence. Results showed that this T base-modified aptamer could still bind PL45 cells with specificity and high affinity. Next, PL45 cells were washed three times with cold PBS buffer and lysed in $5 \mathrm{~mL}$ of hypotonic buffer [50 mM Tris- $\mathrm{HCl}$ (pH7.5) containing protease inhibitors (0.1 mM PMSF and cocktail)] at $4{ }^{\circ} \mathrm{C}$ for $30 \mathrm{~min}$. After centrifugation, the debris was washed three times with $5 \mathrm{~mL}$ of hypotonic buffer and dissolved in $1.5 \mathrm{~mL}$ of lysis buffer (PBS containing 5 $\mathrm{mM} \mathrm{MgCl} 2$ and $1 \%$ Triton $\mathrm{X}-100)$ at $4{ }^{\circ} \mathrm{C}$ for $30 \mathrm{~min}$. The resulting supernatant was incubated with beads, library, or XQ-2d at $4{ }^{\circ} \mathrm{C}$ for $1 \mathrm{~h}$, respectively. Subsequently, the protein-library or protein-XQ-2d complex was captured by incubating it with $2 \mathrm{mg}(200 \mu \mathrm{L})$ of streptavidin beads at $4{ }^{\circ} \mathrm{C}$ for $45 \mathrm{~min}$. After collection by centrifugation, the proteins were eluted by heating in $30 \mu \mathrm{L}$ of loading buffer and analyzed by SDS-PAGE gel and Coomassie blue staining. The aptamer-purified protein bands were excised for digestion in situ and analyzed by LC-MS/MS.

\section{Fluorescence Analysis of Subcellular Localization.}

Cells $\left(1.5 \times 10^{5}\right)$ were seeded in a $35 \mathrm{~mm}$ glass bottom dish and cultured for $24 \mathrm{~h}$. After being washed with cold washing buffer, the cells were incubated with FITC-labeled aptamer and PE-labeled CD71-antibody in $1 \mathrm{~mL}$ of binding buffer at $4{ }^{\circ} \mathrm{C}$ for $1 \mathrm{~h}$. After being washed twice, the cells were imaged by a FV1000-X81 confocal microscope (Olympus, Japan) and analyzed by FV10-ASW Viewer software (version 3.1). 


\section{Aptamer-Based Pull-down Assay.}

The protein samples were prepared in the same manner described for mass spectrometry analysis. The captured proteins were analyzed by SDS-PAGE gel and Coomassie blue staining and subjected to Western blot using anti-CD71 antibody.

\section{RNA Interference.}

Lipofectamine RNAiMAX transfection reagent, the sequence of CD71 siRNA (siRNA1 sense, ACUUGCU-GUAGAUGAAGAA; siRNA2 sense, CUUCCAGACUAACAACA-GA; and a nonspecific siRNA sense, CACGCUCGGUCAAAAG-GUUUU), was purchased from Gene Pharma. PL45 cells were transfected with siRNA by Lipofectamine RNAiMAX transfection reagent for $24 \mathrm{~h}$. The cells then were harvested and analyzed by flow cytometry, and protein extracts were detected by Western blot.

\section{Preparation of XQ-2d-sd3 for DOX Loading.}

The sequences of s1, s2, and XQ-2d+linker were individually snap cooled (heated at $95{ }^{\circ} \mathrm{C}$ for $5 \mathrm{~min}$; incubated on ice for $10 \mathrm{~min}$ ) and then left at room temperature for $30 \mathrm{~min}$. The mixture of $\mathrm{s} 1(10 \mu \mathrm{M})$ and $\mathrm{s} 2(10 \mu \mathrm{M})$ was held at room temperature for $1 \mathrm{~h}$ to form sd3, followed by mixing XQ-2d+linker $(15 \mathrm{uM})$ and $\mathrm{sd} 3(10 \mathrm{uM})$ at room temperature for $1 \mathrm{~h}$ to prepare XQ-2d-sd3. Chimeras were prepared by mixing Dox and prepared XQ-2d-sd3 at room temperature for $1 \mathrm{~h}$ in DPBS supplemented with $\mathrm{MgCl}_{2}(5 \mathrm{mM})$. The fluorescence spectrum of Dox was then examined using a HORIBA Fluoromax-4 instrument $\left(\lambda_{\mathrm{Ex}}=488\right.$ $\left.\mathrm{nm}, \lambda_{\mathrm{Em}}=520-680 \mathrm{~nm}\right)$.

\section{In Vitro Cytotoxicity Assay.}

In vitro cytotoxicity was determined using the CCK-8 assay with 96-well plates. Briefly, cells $\left(6 \times 10^{3}\right.$ cells per well) were treated with XQ-2d-sd3, free drug, or chimeras in medium (without FBS, unless denoted otherwise; $37{ }^{\circ} \mathrm{C} ; 5 \% \mathrm{CO}_{2}$ ). After $4 \mathrm{~h}$, the cells were washed, and fresh medium was added. The cells were then cultured for $48 \mathrm{~h}$, and the CCK-8 assay was used to determine cell viability according to the standard protocol outlined by the manufacturer.

\section{Preparation of Aptamer-Functionalized Gold Nanoparticles (GNPs).}

The strategy for synthesizing the spherical GNPs with different diameters and functionalizing with thiol-modified oligonucleotides followed our previous published work on surface energy transfer nanoruler. ${ }^{17}$ The $5^{\prime}$-thiol-modified DNA (XQ-2d aptamer and Lib sequences) with a spacer of five T bases was purchased from Sangon Biotech (Shanghai) Co., Ltd. In brief, the surface area for each size of GNPs was calculated, and the thiolated DNA molecules were mixed with GNP sols with a ratio of 5 DNA molecules per $\mathrm{nm}^{2}$ for

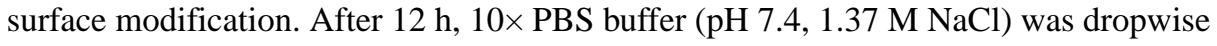
added to the reaction mixture to elevate the $\mathrm{NaCl}$ concentration to $0.05 \mathrm{M}$. After another 12 $\mathrm{h}$, a similar procedure was performed to elevate to a final $\mathrm{NaCl}$ concentration of $0.1 \mathrm{M}$. The mixture was stirred for a third $12 \mathrm{~h}$. A twice washing procedure was performed to remove unbound DNA by centrifugation $(10000 g)$ and resuspension. The GNP@DNA probe was 
resuspended in a $1 \times$ PBS solution ( $\mathrm{pH}$ 7.4) with a final concentration of $20 \mathrm{nM}$ and used for UV-vis absorption measurements. ${ }^{33}$

\section{Structures of Protein and Aptamer.}

The protein structure of CD71 was obtained from the RCSB PDB databank (PDB ID: $3 \mathrm{~S} 9 \mathrm{~L})^{34}$ with a high resolution of $3.22 \AA$. Water molecules, cocrystallized ligand, and ions in protein complex were removed for the docking process. The Vienna number of aptamer (XQ-2d: ACTCATAGGGTTAGGGGCTGCTGGCCAGATACTCA-

GATGGTAGGGTTACTATGAGC) was online formed by the MFOLD Web server, ${ }^{35}$ and the corresponding three-dimensional structure with best predicted energy was constructed using the RNAcomposer software. ${ }^{36}$ After nucleic acid bases $\mathrm{U}$ were changed to T, one positionconstrained molecular dynamics simulation was performed to eliminate the steric conflicts within the aptamer system.

\section{Molecular Docking Methods.}

The following three steps (blind docking and precise docking) were performed to explore possible binding interface between CD71 and the aptamer. Docking simulations were performed with Rosetta ${ }^{37}$ software. (1) The docking protocol began with a rigid-body blind docking in which all heavy atoms of protein and aptamer systems were strictly position restrained. In this step, we randomly placed the protein and aptamer roughly facing each other, and at least 2000 docking conformations were obtained for further analysis. Amino acids forming hydrogen bonds between protein and aptamer in each docking conformation were collected and totaled. Finally, the amino acid with high ratio (>0.4) that participated in $\mathrm{H}$-bonds was selected as potential binding spot. Binding spots far away from the others were manually deleted, and the rest were regarded as the final spots. Using this approach, we were able to achieve an acceptable-quality prediction for aptamer "binding pocket". (2) Rosetta software is most accurate when docking locally. In precise docking, the aptamer was randomly placed (within $\sim 10 \AA$ ) within the binding pockets, and then the input structure of aptamer was perturbed by $3 \AA$ translation and $8^{\circ}$ rotation before the start of every individual simulation. During precise docking, the side chains of the protein residues at the binding pocket and the complete DNA (excluding terminal base pairs) were allowed to move. Finally, a maximum number of 100 conformers were considered, and the conformation with the lowest binding energy was selected for final molecular dynamics simulations.

\section{Molecular Dynamics Simulations.}

MD simulations was carried out by AMBER16 software ${ }^{38,39}$ based on the best docking conformation of CD71-XQ-2d complex. Before the simulation, hydrogen atoms were added to initialize the complex using the leap module, and default protonation states at neutral value were set to ionize the amino acids. The program AmberTools with AMBER ff99sb force field and the TIP3P water model were performed to generate complex topology files. $\mathrm{Na}^{+}$and $\mathrm{Cl}^{-}$were added to make the complex system neutral, the final salt concentration of which was $0.15 \mathrm{M}$. Under periodic boundary conditions with particle mesh Ewald (PME) method, the molecular dynamics simulation was executed with a minimal distance of $0.1 \mathrm{~nm}$ to the margin of the box. Bond lengths were all constrained, and the integration time step was set to 2 fs using the Verlet leapfrog algorithm. 
The entire system was first minimized to eliminate possible conflicts between the solute and the solvent. CD71-XQ-2d complex was subsequently restrained with a harmonic potential as the form $k(\Delta x)^{2}$ force constant was set to $k=100 \mathrm{kcal} / \mathrm{mol}^{-1} \cdot \AA^{-2}$. Utilizing the steepest descent and conjugate gradient minimization algorithm for water and counterions made the entire system energy minimize. The complex system then reached equilibrium with the 500 ps NVT simulation for the complex and the maximum force limited to $1000 \mathrm{kcal} / \mathrm{mol} / \mathrm{nm}$. After that, the heating phase was executed with no restrain on heavy atoms gradually in the NPT ensemble from 0 to $298 \mathrm{~K}$ over 500 ps. Finally, we performed a 10 ns MD simulation with a $2000 \mathrm{ps}$ time step under the condition of $298 \mathrm{k}$ and 1 atm for protein-aptamer complexes. A total number of 3000 snapshots extracted from last 3 ns MD simulation trajectory was used for the final average structure of CD71-XQ-2d complex.

\section{Supplementary Material}

Refer to Web version on PubMed Central for supplementary material.

\section{ACKNOWLEDGMENTS}

This work was supported by grants from the National Natural Science Foundation of China (nos. 21521063, 21890744, and 81672760), the Natural Science Foundation of Hunan Province (2016JJ3048), and the Hunan Provincial Key Research and Development Plan (2018SK2128). This work is also supported by NIH GM R35 127130 and NSF 1645215.

\section{REFERENCES}

(1). Siegel RL; Miller KD; Jemal A Cancer Statistics, 2017. Ca-Cancer J. Clin 2017, 67 (1), 7-30. [PubMed: 28055103]

(2). Hidalgo M Pancreatic cancer. N. Engl. J. Med 2010, 362 (17), 1605-17. [PubMed: 20427809]

(3). Khan MA; Zubair H; Srivastava SK; Singh S; Singh AP Insights into the Role of microRNAs in Pancreatic Cancer Pathogenesis: Potential for Diagnosis, Prognosis, and Therapy. Adv. Exp. Med. Biol 2015, 889, 71-87. [PubMed: 26658997]

(4). Ye M; Hu J; Peng M; Liu J; Liu J; Liu H; Zhao X; Tan W Generating aptamers by cell-SELEX for applications in molecular medicine. Int. J. Mol. Sci 2012, 13 (3), 3341-53. [PubMed: 22489154]

(5). Strehlitz B; Reinemann C; Linkorn S; Stoltenburg R Aptamers for pharmaceuticals and their application in environmental analytics. Bioanal Rev. 2012, 4 (1), 1-30. [PubMed: 22389661]

(6). Zhang H; Wang Z; Xie L; Zhang Y; Deng T; Li J; Liu J; Xiong W; Zhang L; Zhang L; Peng B; He L; Ye M; Hu X; Tan W Molecular Recognition and In-Vitro-Targeted Inhibition of Renal Cell Carcinoma Using a DNA Aptamer. Mol. Ther.-Nucleic Acids 2018, 12, 758-768. [PubMed: 30141409]

(7). Zhou J; Rossi JJ Cell-specific aptamer-mediated targeted drug delivery. Oligonucleotides 2011, 21 (1), 1-10. [PubMed: 21182455]

(8). Lyu Y; Chen G; Shangguan D; Zhang L; Wan S; Wu Y; Zhang H; Duan L; Liu C; You M Generating cell targeting aptamers for nanotheranostics using cell-SELEX. Theranostics 2016, 6 (9), 1440. [PubMed: 27375791]

(9). Duan M; Long Y; Yang C; Wu X; Sun Y; Li J; Hu X; Lin W; Han D; Zhao Y; Liu J; Ye M; Tan W Selection and characterization of DNA aptamer for metastatic prostate cancer recognition and tissue imaging. Oncotarget 2016, 7 (24), 36436-36446. [PubMed: 27183906]

(10). Wang Y; Luo Y; Bing T; Chen Z; Lu M; Zhang N; Shangguan D; Gao X DNA aptamer evolved by cell-SELEX for recognition of prostate cancer. PLoS One 2014, 9 (6), e100243. [PubMed: 24956390] 
(11). Van Simaeys D; Lopez-Colon D; Sefah K; Sutphen R; Jimenez E; Tan W Study of the molecular recognition of aptamers selected through ovarian cancer cell-SELEX. PLoS One 2010, 5 (11), e13770. [PubMed: 21072169]

(12). Shangguan D; Cao Z; Meng L; Mallikaratchy P; Sefah K; Wang H; Li Y; Tan W Cell-specific aptamer probes for membrane protein elucidation in cancer cells. J. Proteome Res 2008, 7 (5), 2133-9. [PubMed: 18363322]

(13). Mallikaratchy P; Tang Z; Kwame S; Meng L; Shangguan D; Tan W Aptamer directly evolved from live cells recognizes membrane bound immunoglobin heavy mu chain in Burkitt's lymphoma cells. Mol. Cell. Proteomics 2007, 6 (12), 2230-8. [PubMed: 17875608]

(14). Daniels DA; Chen H; Hicke BJ; Swiderek KM; Gold L A tenascin-C aptamer identified by tumor cell SELEX: systematic evolution of ligands by exponential enrichment. Proc. Natl. Acad. Sci. U. S. A 2003, 100 (26), 15416-21. [PubMed: 14676325]

(15). Van Simaeys D; Turek D; Champanhac C; Vaizer J; Sefah K; Zhen J; Sutphen R; Tan W Identification of cell membrane protein stress-induced phosphoprotein 1 as a potential ovarian cancer biomarker using aptamers selected by cell systematic evolution of ligands by exponential enrichment. Anal. Chem 2014, 86 (9), 4521-7. [PubMed: 24654750]

(16). Wu X; Zhao Z; Bai H; Fu T; Yang C; Hu X; Liu Q; Champanhac C; Teng IT; Ye M; Tan W DNA Aptamer Selected against Pancreatic Ductal Adenocarcinoma for in vivo Imaging and Clinical Tissue Recognition. Theranostics 2015, 5 (9), 985-94. [PubMed: 26155314]

(17). Chen Y; O’Donoghue MB; Huang YF; Kang H; Phillips JA; Chen X; Estevez MC; Yang CJ; Tan W A surface energy transfer nanoruler for measuring binding site distances on live cell surfaces. J. Am. Chem. Soc 2010, 132 (46), 16559-70. [PubMed: 21038856]

(18). Yun CS; Javier A; Jennings T; Fisher M; Hira S; Peterson S; Hopkins B; Reich NO; Strouse GF Nanometal surface energy transfer in optical rulers, breaking the FRET barrier. J. Am. Chem. Soc 2005, 127 (9), 3115-9. [PubMed: 15740151]

(19). Goossens N; Nakagawa S; Sun X; Hoshida Y Cancer biomarker discovery and validation. Transl. Cancer Res 2015, 4, 256-269. [PubMed: 26213686]

(20). Berezovski MV; Lechmann M; Musheev MU; Mak TW; Krylov SN Aptamer-facilitated biomarker discovery (AptaBiD). J. Am. Chem. Soc 2008, 130 (28), 9137-43. [PubMed: 18558676]

(21). Rychtarcikova Z; Lettlova S; Tomkova V; Korenkova V; Langerova L; Simonova E; Zjablovskaja P; Alberich-Jorda M; Neuzil J; Truksa J Tumor-initiating cells of breast and prostate origin show alterations in the expression of genes related to iron metabolism. Oncotarget 2017, 8 (4), 63766398. [PubMed: 28031527]

(22). Chua AC; Klopcic BR; Ho DS; Fu SK; Forrest CH; Croft KD; Olynyk JK; Lawrance IC; Trinder D Dietary iron enhances colonic inflammation and IL-6/IL-11-Stat3 signaling promoting colonic tumor development in mice. PLoS One 2013, 8 (11), e78850. [PubMed: 24223168]

(23). Chan KT; Choi MY; Lai KK; Tan W; Tung LN; Lam HY; Tong DK; Lee NP; Law S Overexpression of transferrin receptor CD71 and its tumorigenic properties in esophageal squamous cell carcinoma. Oncol. Rep 2014, 31 (3), 1296-304. [PubMed: 24435655]

(24). Jeong SM; Hwang S; Seong RH Transferrin receptor regulates pancreatic cancer growth by modulating mitochondrial respiration and ROS generation. Biochem. Biophys. Res. Commun 2016, 471 (3), 373-9. [PubMed: 26869514]

(25). Jeong SM; Lee J; Finley LW; Schmidt PJ; Fleming MD; Haigis MC SIRT3 regulates cellular iron metabolism and cancer growth by repressing iron regulatory protein 1 . Oncogene 2015, 34 (16), 2115-24. [PubMed: 24909164]

(26). Ryschich E; Huszty G; Knaebel HP; Hartel M; Buchler MW; Schmidt J Transferrin receptor is a marker of malignant phenotype in human pancreatic cancer and in neuroendocrine carcinoma of the pancreas. Eur. J. Cancer 2004, 40 (9), 1418-22. [PubMed: 15177502]

(27). Jhaveri DT; Kim MS; Thompson ED; Huang L; Sharma R; Klein AP; Zheng L; Le DT; Laheru DA; Pandey A; Jaffee EM; Anders RA Using Quantitative Seroproteomics to Identify Antibody Biomarkers in Pancreatic Cancer. Cancer Immunol. Res 2016, 4 (3), 225-33. [PubMed: 26842750] 
(28). Aisen P Transferrin receptor 1. Int. J. Biochem. Cell Biol 2004, 36 (11), 2137-43. [PubMed: 15313461]

(29). Cheng Y; Zak O; Aisen P; Harrison SC; Walz T Structure of the human transferrin receptortransferrin complex. Cell 2004, 116 (4), 565-76. [PubMed: 14980223]

(30). Cabantchik ZI Labile iron in cells and body fluids: physiology, pathology, and pharmacology. Front. Pharmacol 2014, 5, 45. [PubMed: 24659969]

(31). Basuli D; Tesfay L; Deng Z; Paul B; Yamamoto Y; Ning G; Xian W; McKeon F; Lynch M; Crum CP; Hegde P; Brewer M; Wang X; Miller LD; Dyment N; Torti FM; Torti SV Iron addiction: a novel therapeutic target in ovarian cancer. Oncogene 2017, 36 (29), 4089-4099. [PubMed: 28319068]

(32). Singh M; Mugler K; Hailoo DW; Burke S; Nemesure B; Torkko K; Shroyer KR Differential expression of transferrin receptor (TfR) in a spectrum of normal to malignant breast tissues: implications for in situ and invasive carcinoma. Appl. Immunohistochem Mol. Morphol 2011, 19 (5), 417-23. [PubMed: 21297444]

(33). Tortorella S; Karagiannis TC Transferrin receptor-mediated endocytosis: a useful target for cancer therapy. J. Membr. Biol 2014, 247 (4), 291-307. [PubMed: 24573305]

(34). Haiss W; Thanh NT; Aveyard J; Fernig DG Determination of size and concentration of gold nanoparticles from UV-vis spectra. Anal. Chem 2007, 79 (11), 4215-21. [PubMed: 17458937]

(35). Eckenroth BE; Steere AN; Chasteen ND; Everse SJ; Mason AB How the binding of human transferrin primes the transferrin receptor potentiating iron release at endosomal $\mathrm{pH}$. Proc. Natl. Acad. Sci. U. S. A 2011, 108 (32), 13089-94. [PubMed: 21788477]

(36). Zuker M Mfold web server for nucleic acid folding and hybridization prediction. Nucleic Acids Res. 2003, 31 (13), 3406-15. [PubMed: 12824337]

(37). Biesiada M; Purzycka KJ; Szachniuk M; Blazewicz J; Adamiak RW Automated RNA 3D Structure Prediction with RNAComposer. Methods Mol. Biol 2016, 1490, 199-215. [PubMed: 27665601]

(38). Gray JJ; Moughon S; Wang C; Schueler-Furman O; Kuhlman B; Rohl CA; Baker D Proteinprotein docking with simultaneous optimization of rigid-body displacement and side-chain conformations. J. Mol. Biol 2003, 331 (1), 281-99. [PubMed: 12875852]

(39). Case DA; Cheatham TE 3rd; Darden T; Gohlke H; Luo R; Merz KM Jr.; Onufriev A; Simmerling C; Wang B; Woods RJ The Amber biomolecular simulation programs. J. Comput. Chem 2005, 26 (16), 1668-88. [PubMed: 16200636] 
$\mathbf{A}$

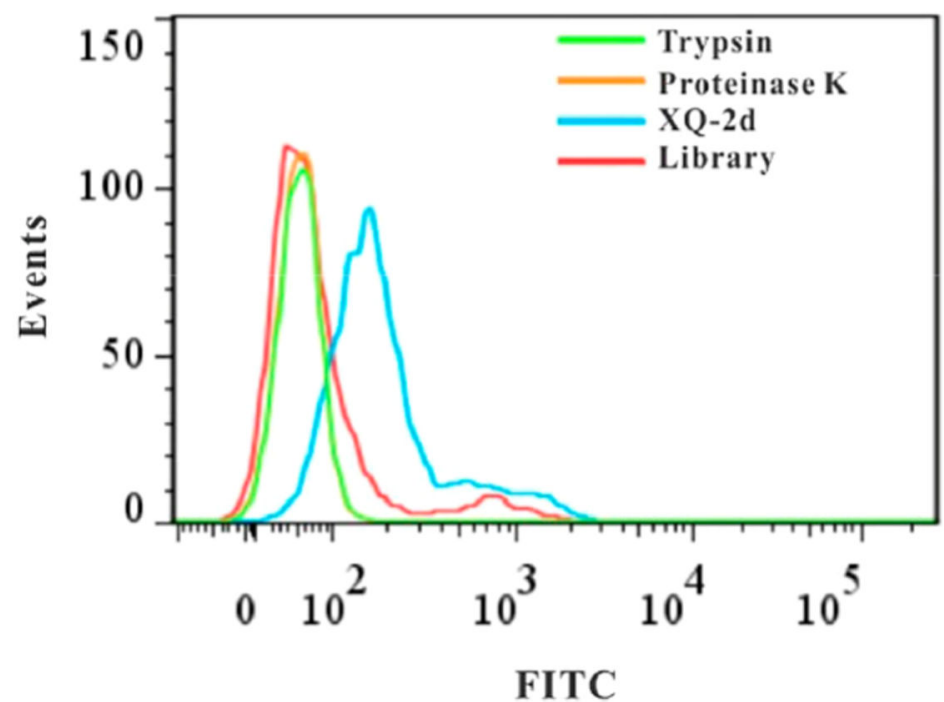

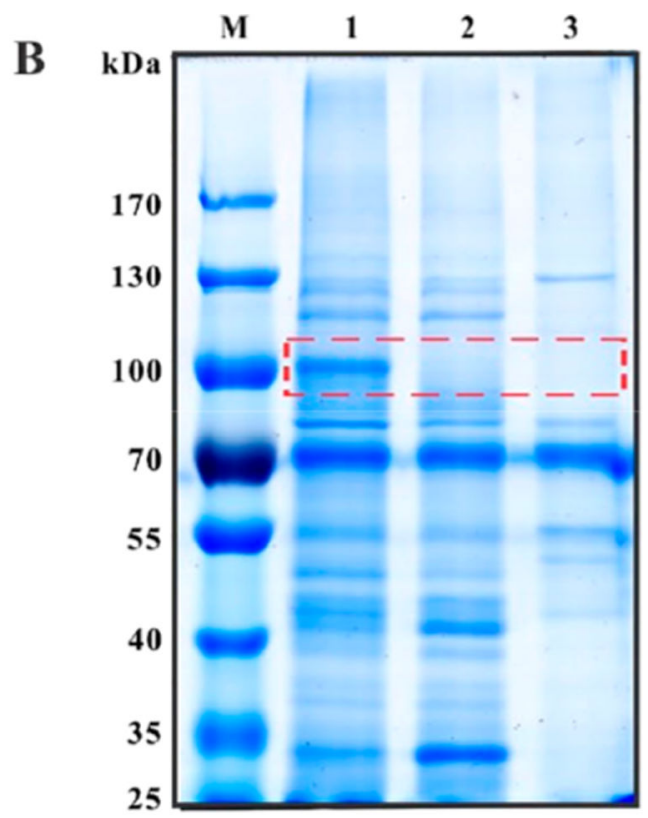

Figure 1.

XQ-2d-binding proteins were analyzed by mass spectrometry. (A) After treatment with trypsin and Proteinase K, the binding of XQ-2d to PL45 cells was analyzed by flow cytometry. (B) Coomassie blue-stained SDS-PAGE was used to analyze aptamer-assisted target purification. $\mathrm{M}$, molecular markers; 1 , protein captured with XQ-2d; 2, protein captured with the library sequences; 3 , magnetic beads only. 
A

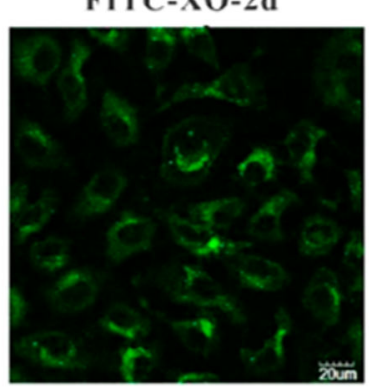

Merge

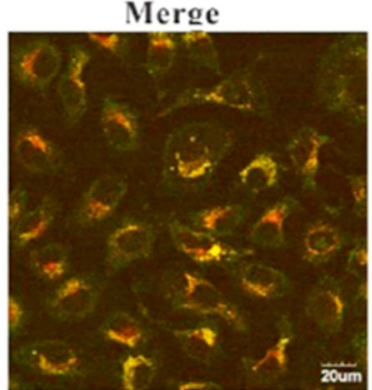

D
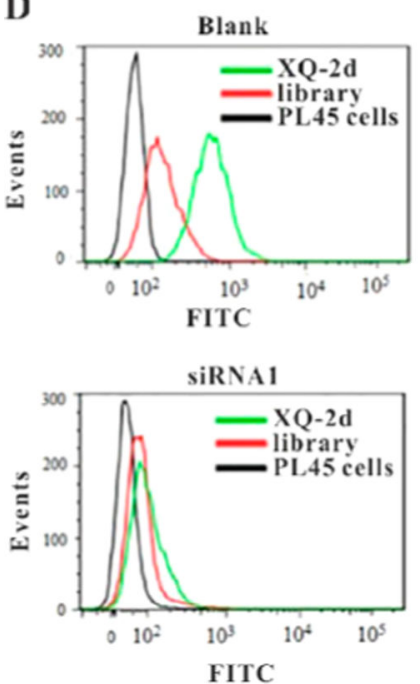

PE-CD71

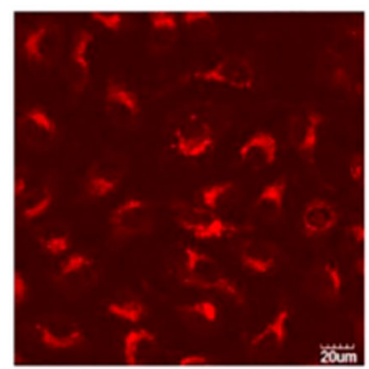

DIC
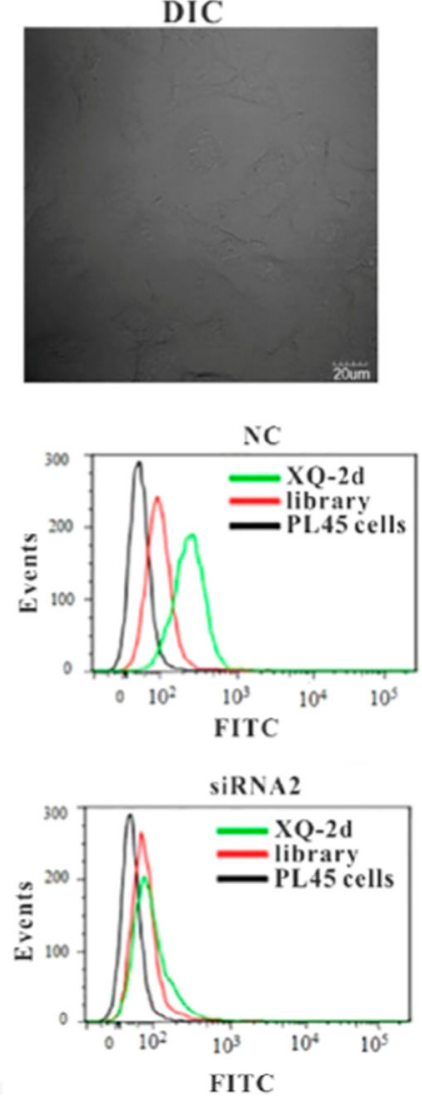

B

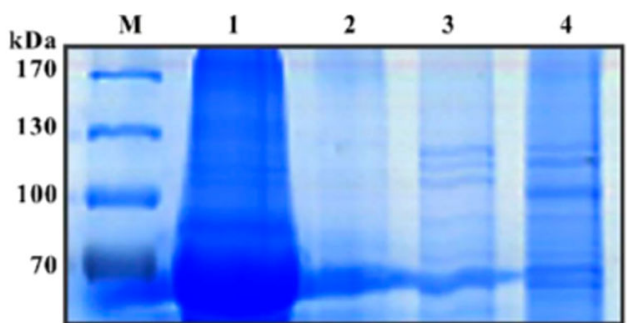

CD71

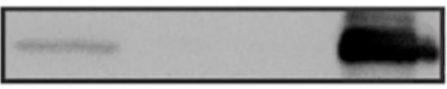

C

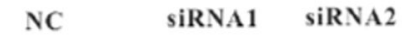

CD71

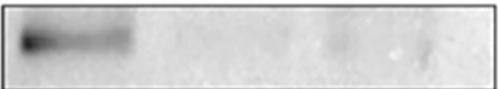

$\beta$-actin

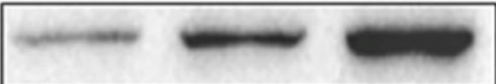

E

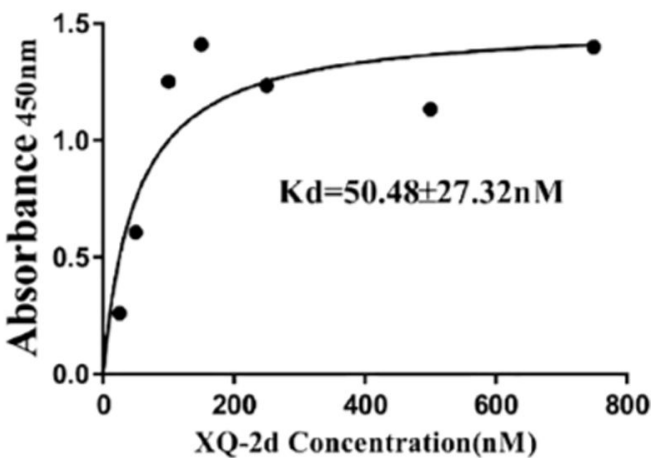

Figure 2.

CD71 is identified as a target for XQ-2d. (A) The subcellular localization of XQ-2d and CD71 in PL45 cells was visualized using FITC-labeled XQ-2d and PE-labeled anti-CD71 antibody. (B) Membrane proteins from PL45 cells (lane 1) were incubated with beads (lane 2), beads conjugated with library (lane 3 ), and beads conjugated with XQ-2d (lane 4). The bound proteins were eluted and analyzed by Western blotting using anti-CD71 antibody. (C) PL45 cells were transfected with the indicated siRNAs. The resulting cell extracts were analyzed using Western blot with anti-CD71 antibody. (D) After PL45 cells were treated with the indicated siRNAs, the binding of XQ-2d to PL45 cells was analyzed by flow cytometry. (E) Dissociation constant of XQ-2d for CD71 was determined by ELISA. 
A

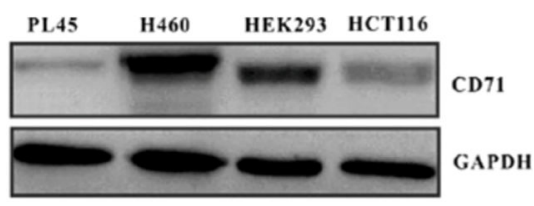

C
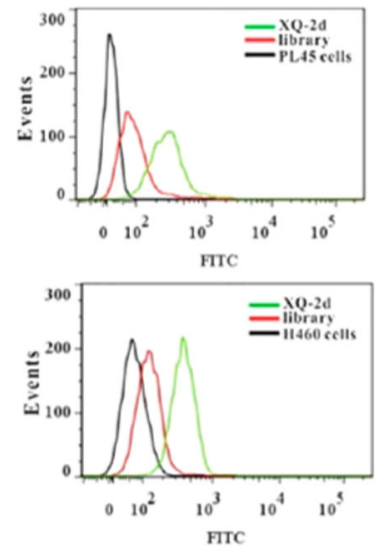

E

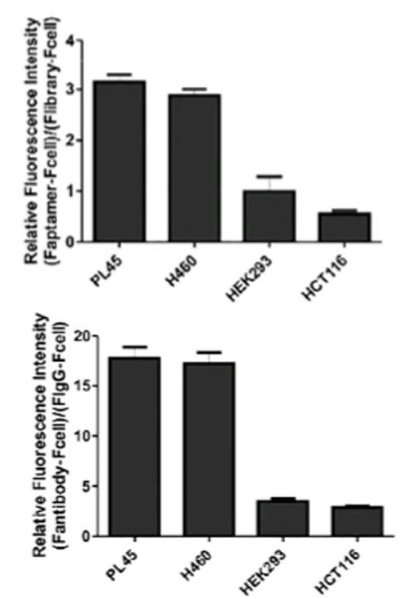

B

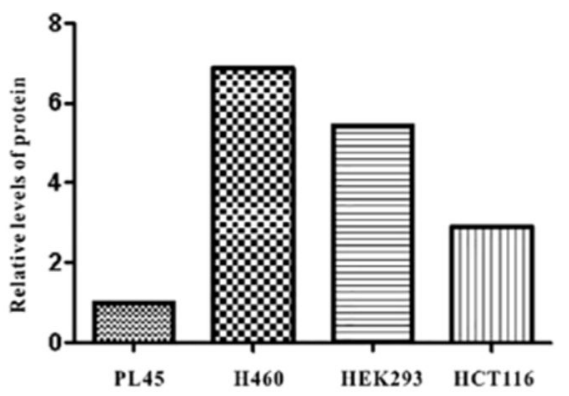

D
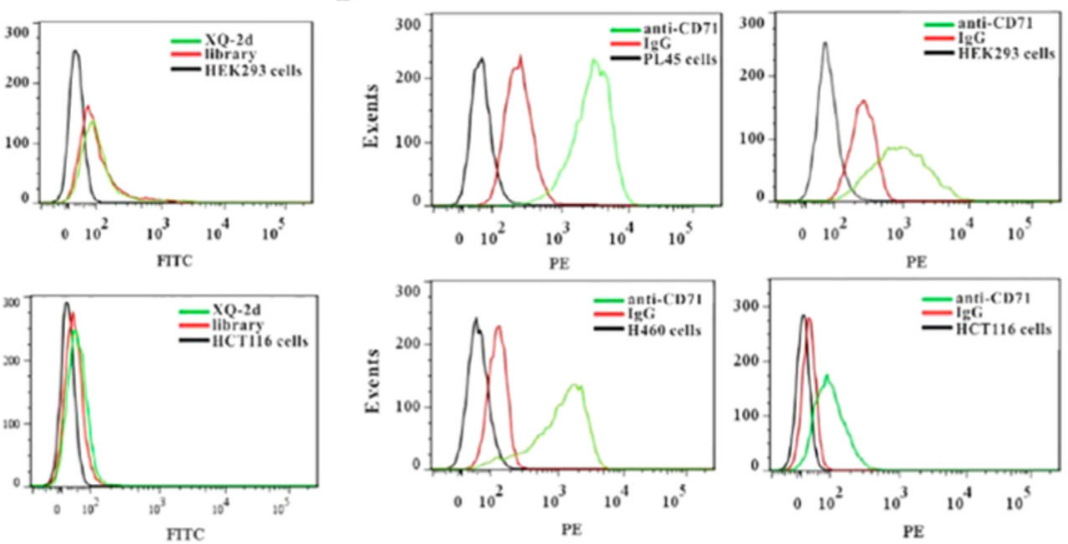

F
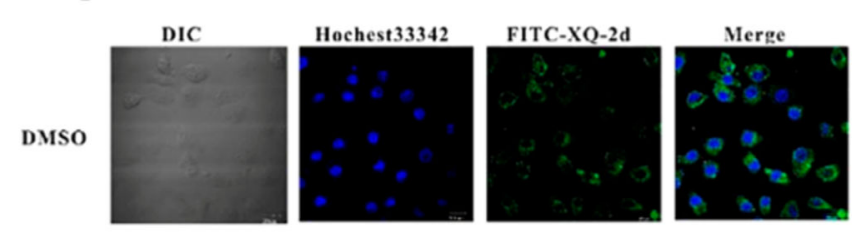

Dynasore
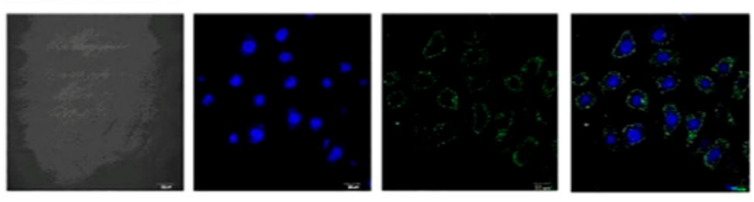

\section{Figure 3.}

Membrane-bound CD71 is the target of XQ-2d. (A) Total proteins from the indicated cell lines were extracted and subjected to Western blot using anti-CD71 or anti-GAPDH antibody. (B) Quantification of CD71 levels relative to GAPDH expression was shown. (C) The binding of XQ-2d to the indicated cell lines was analyzed by flow cytometry. (D) CD71 expression on the membrane of the indicated cell lines was analyzed using anti-CD71 antibody by flow cytometry. (E) Relative fluorescent intensities of XQ-2d and CD71 are shown. (F) After PL45 cells were treated with Dynasore, the binding of XQ-2d to PL45 cells was detected by confocal microscopy. The nucleus was stained with Hoechst 33342. 
A

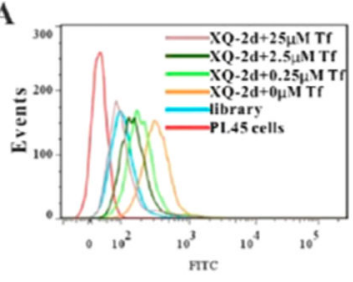

B

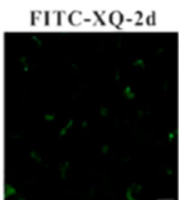

TF+FITC-XO-2
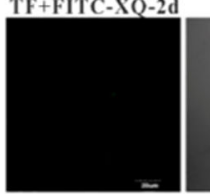

DIC

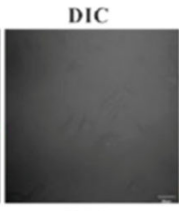

섬

$$
\text { F }
$$

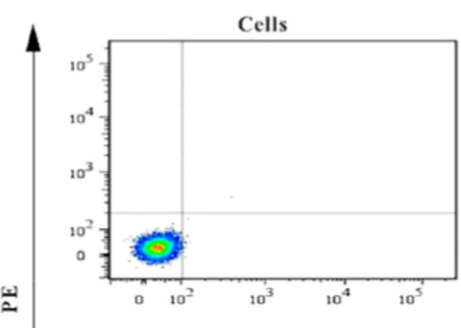

PE-anti-CD71

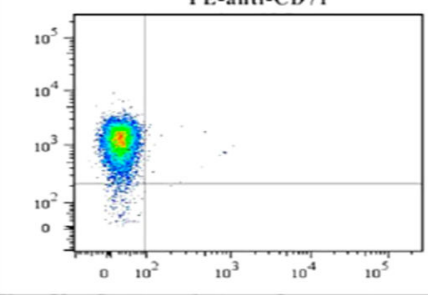

C

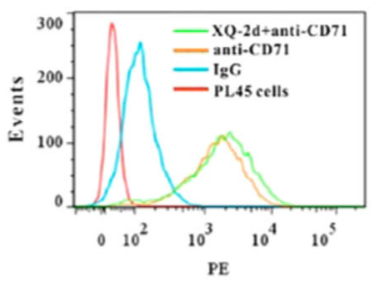

D

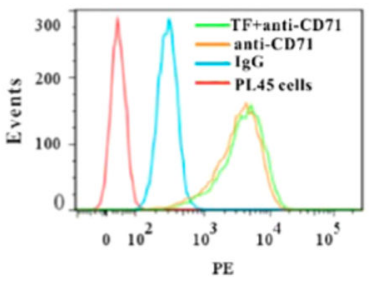

E

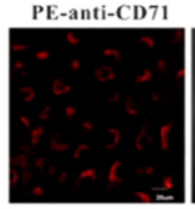

TF+PE-anti-CD71

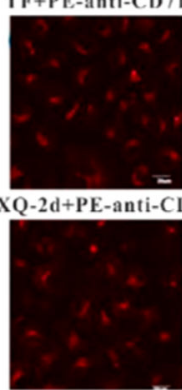

FITC-library

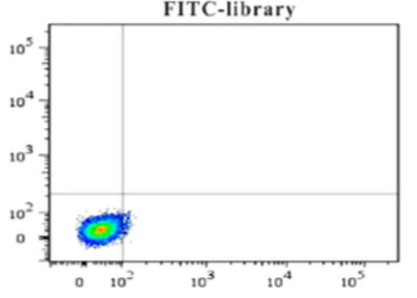

FITC-library+PE-anti-CD 71

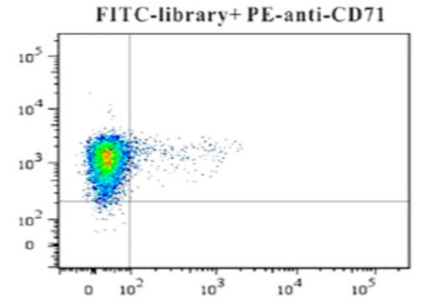

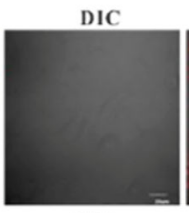

DIC

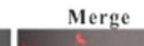

Merge
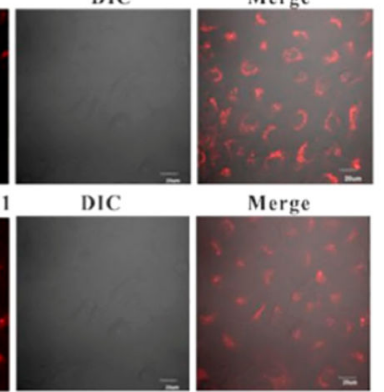

Merge

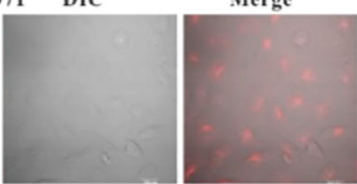

FITC-XQ2d
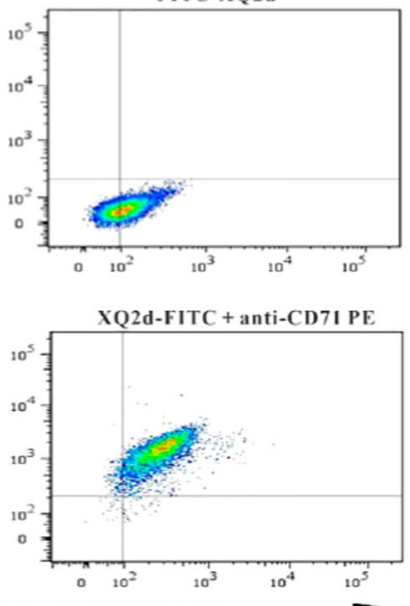

FITC

Figure 4.

XQ-2d shares the same binding site on CD71 with transferrin, but not anti-CD71 antibody.

(A) PL45 cells were preincubated with FITC-labeled XQ-2d (250 nM) before being incubated with the indicated concentration of transferrin. Competition binding was analyzed by flow cytometry. (B) PL45 cells were incubated with FITC-labeled XQ-2d or preincubated with unlabeled transferrin before being incubated with FITC-labeled XQ-2d. The binding of XQ-2d to PL45 cells was analyzed by confocal microscopy. (C and D) PL45 cells were preincubated with XQ-2d (C) or transferrin (D) before being incubated with PE-labeled antiCD71 antibody. The binding of anti-CD71 antibody to PL45 cells was analyzed by flow cytometry. (E) PL45 cells were incubated with PE-labeled anti-CD71 antibody or preincubated with unlabeled Tf or XQ-2d before being incubated with PE-labeled anti-CD71 
antibody. The binding of anti-CD71 antibody to PL45 cells was analyzed by confocal microscopy. (F) PL45 cells were stained with FITC-labeled library, FITC-labeled XQ-2d-, and/or PE-labeled anti-CD71 anti-body. The fluorescent signals from FITC and PE were analyzed by flow cytometry. 
A

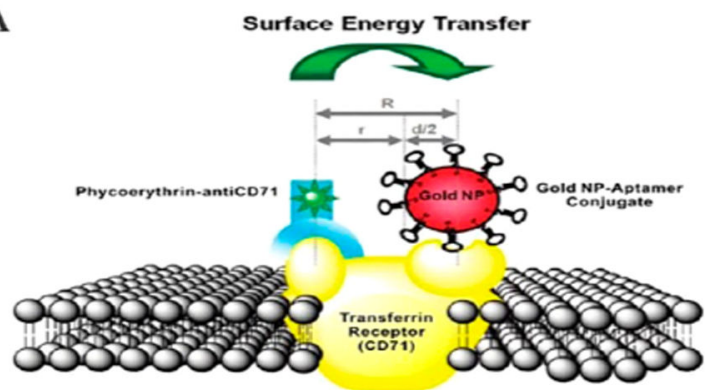

C

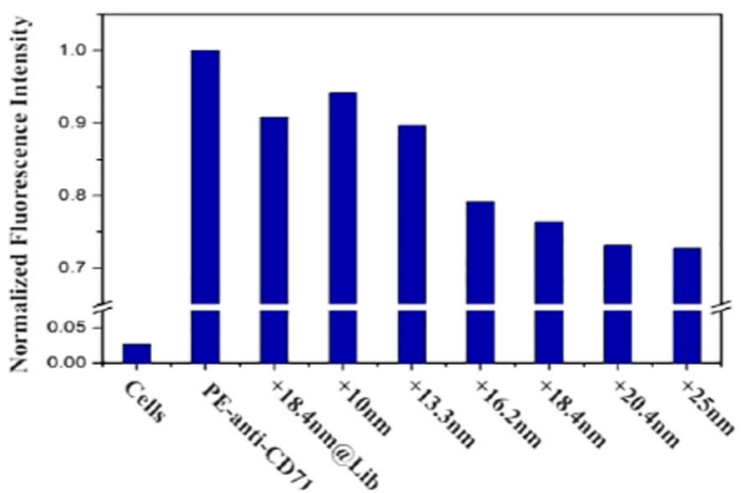

$\mathbf{F}$

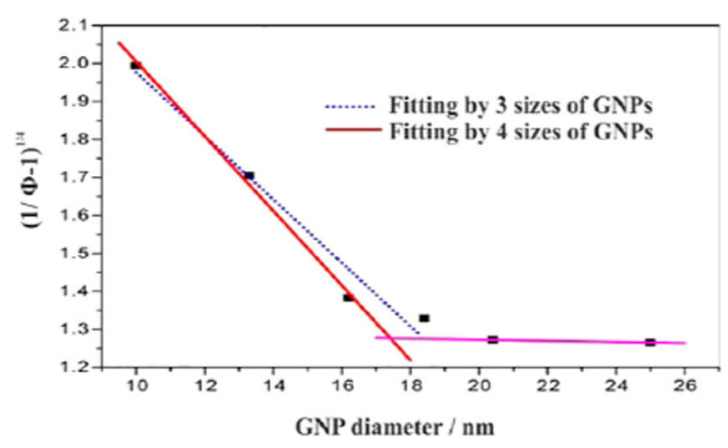

B

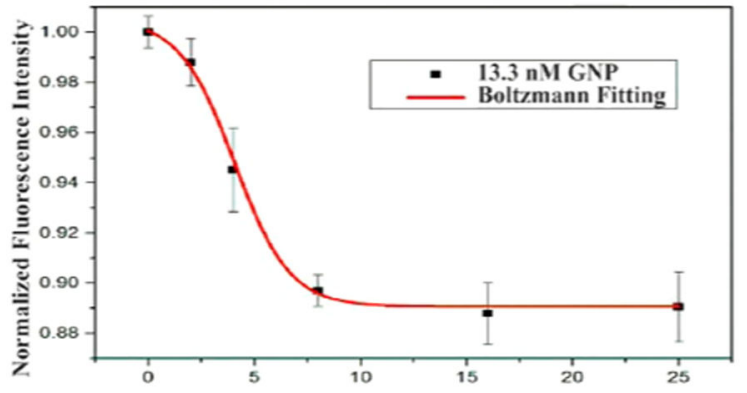

D

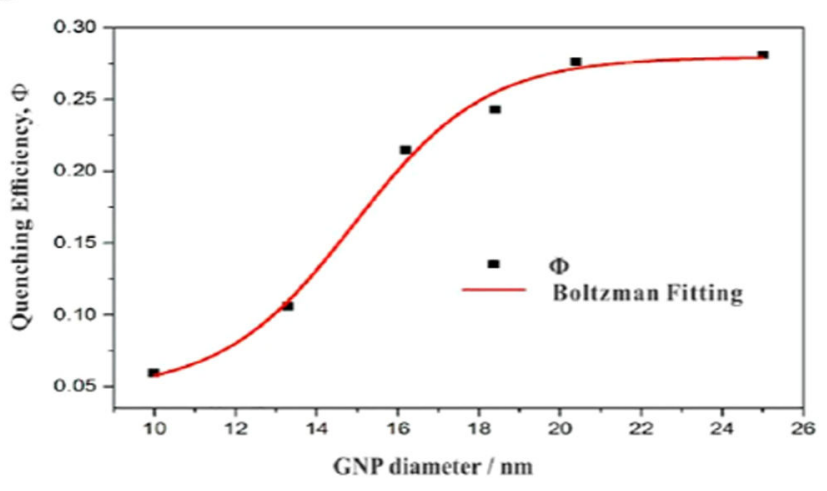

Figure 5.

SET nanoruler for measuring binding site distances on PL45 cell surfaces. (A) A working model for the SET Nanoruler. (B) Determination of saturation binding of aptamer-GNP conjugates on PL45 cells. (C) Histogram of the normalized mean fluorescence intensity for the fluorescence quenching assay determined from the flow cytometry results. The concentration of GNPs was $8 \mathrm{nM}$. (D) Relationship between fluorescence quenching efficiency and GNP diameter. (E) Binding site distance determination. 
A

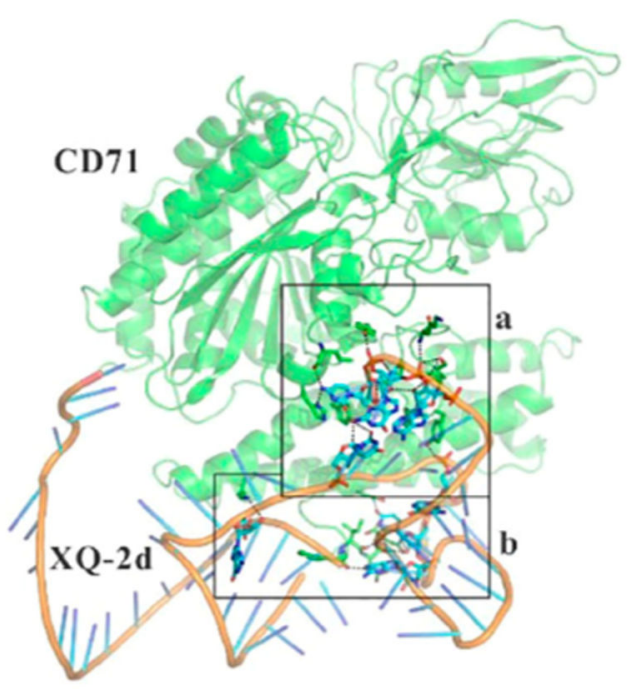

C

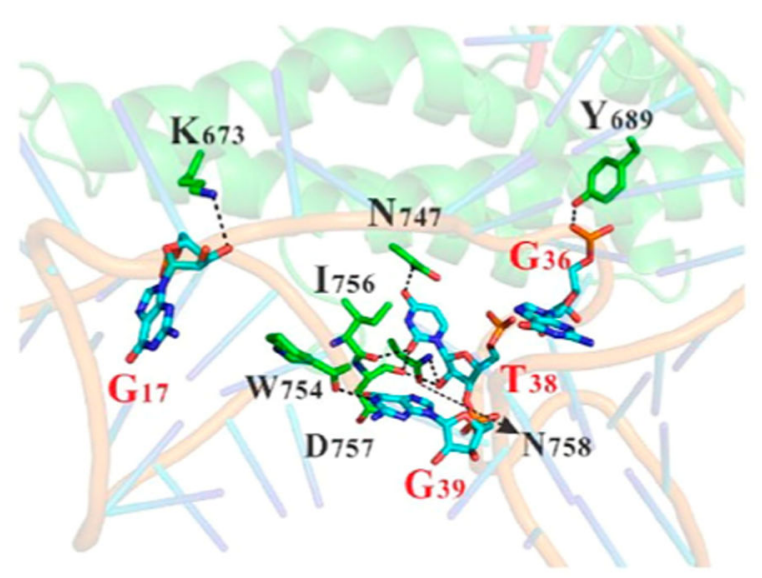

B

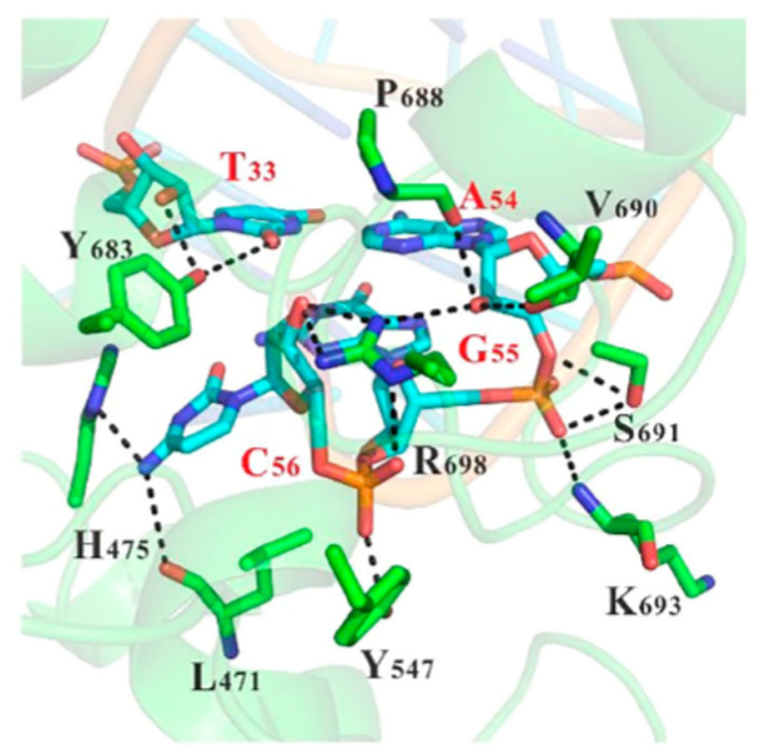

D

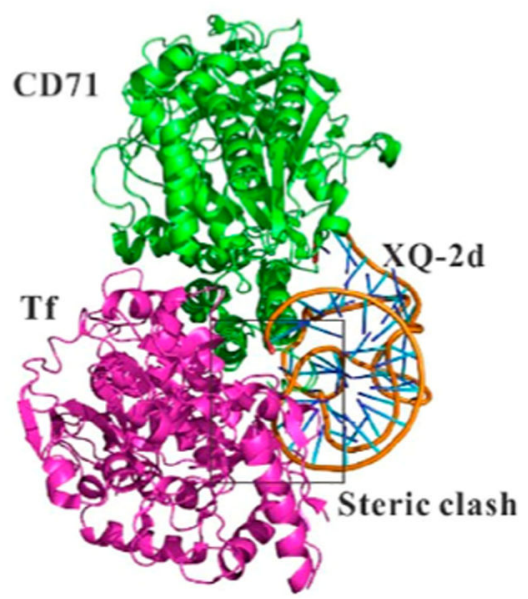

Figure 6.

Identification of interaction between CD71 and XQ-2d. (A) Interaction model between CD71 and XQ-2d. (B and C) Close view of CD71 and XQ-2d interaction region in part a (B) and part b (C). The key residues are marked in black as follows: L471, H475, Y547, K673, Y683, P688, Y689, S691, K693, R698, N747, W754, I756, D757, N758, V690. (D) Conformation superposition of crystal CD71, Tf, and XQ-2d. 
A

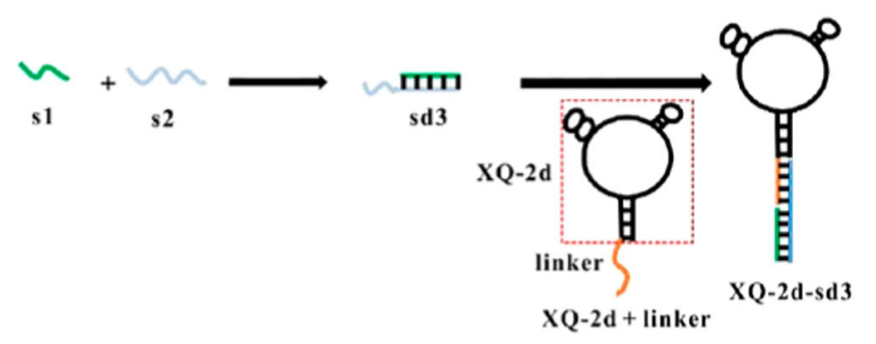

C

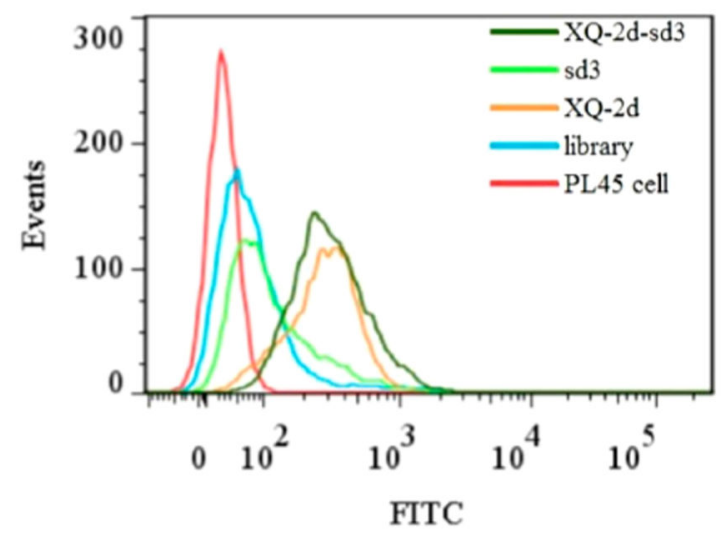

B

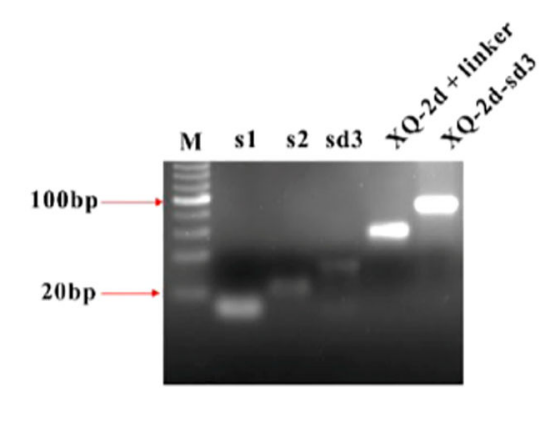

D

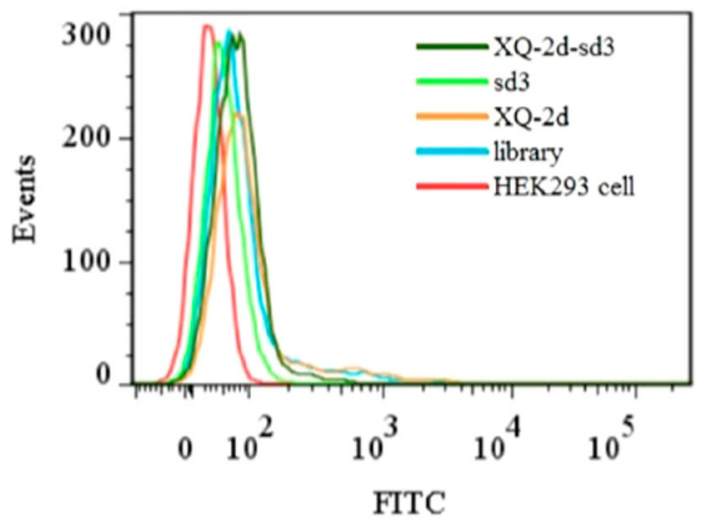

E
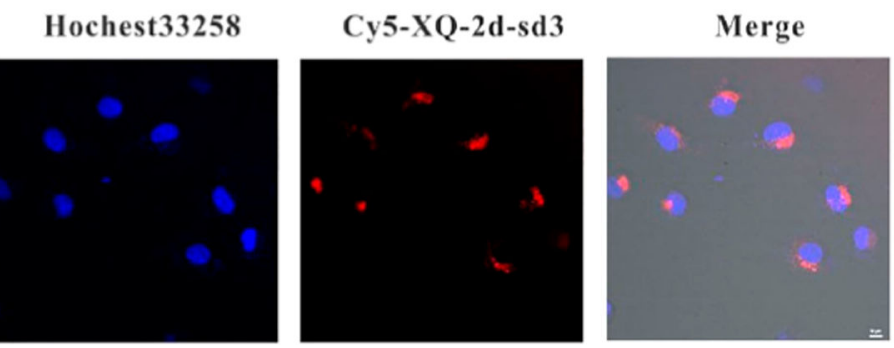

Figure 7.

XQ-2d self-assembles into GC-enriched XQ-2d-sd3. (A) Schematic diagram of XQ-2d-sd3. (B) The assembly of XQ-2d-sd3 was analyzed by agarose gel electrophoresis. (C and D) The binding of library, XQ-2d, sd3, and XQ-2d-sd3 to PL45 cells (C) or HEK293 cells (D) was analyzed by flow cytometry. (E) The internalization of Cy5-labeled XQ-2d-sd3 was detected by confocal microscopy. 


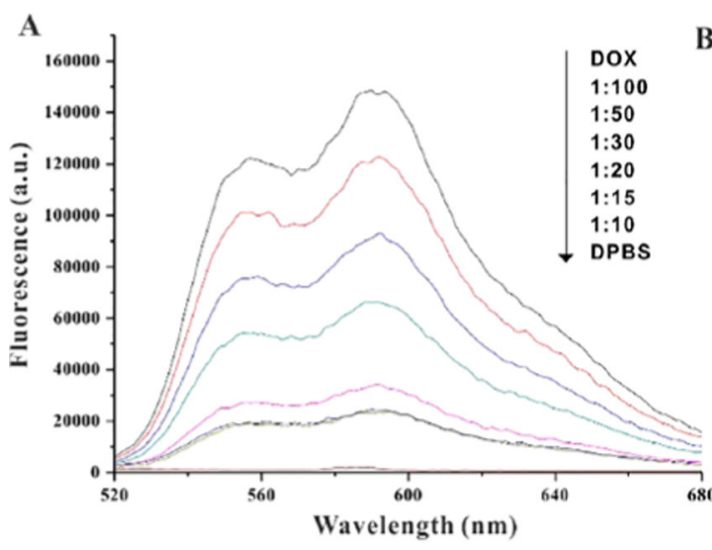

C

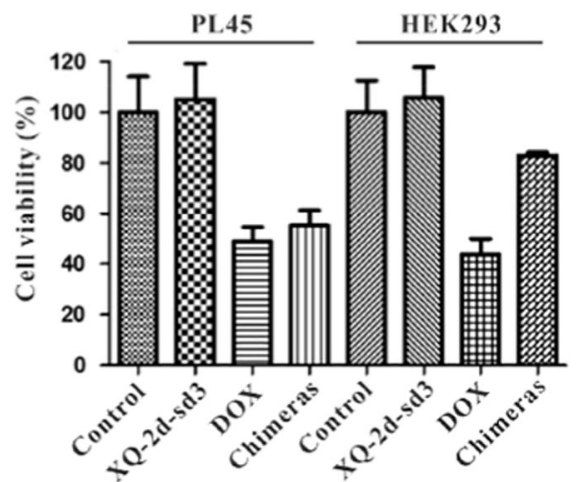

Figure 8. cells or HEK293 cells was analyzed by CCK-8 assay.
B

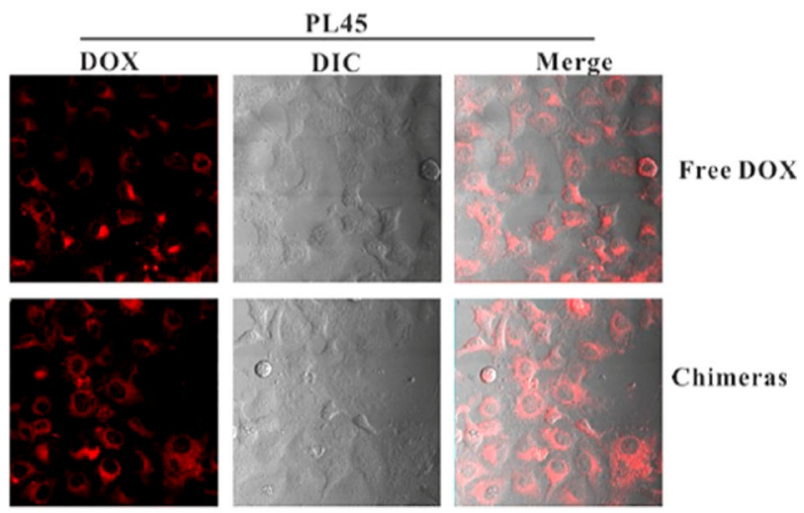

D

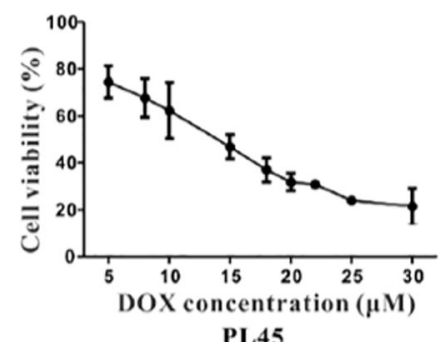

Chimeras

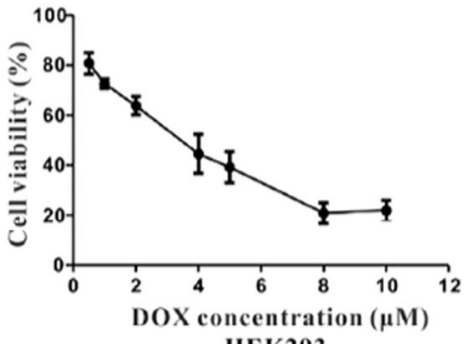

HEK293

XQ-2d-sd3 specifically delivers Dox into PL45 cells. (A) Fluorescence spectra of Dox with increasing equivalents of $\mathrm{XQ}-2 \mathrm{~d}-\mathrm{sd} 3$ (shown by values from top to bottom). (B and $\mathrm{C}$ ) After PL45 cells (B) or HEK293 cells (C) were incubated with free Dox or chimeras, the intracellular signaling of Dox was detected by confocal microscopy. (D) After PL45 cells or HEK293 cells were treated with the indicated concentration of Dox, cell viability was measured by CCK-8 assay. (E) The cytotoxicity of XQ-2d-sd3, Dox, and chimeras on PL45 